Geometry 8 Topology

Volume 9 (2005) 1881-1913

Published: 6 October 2005

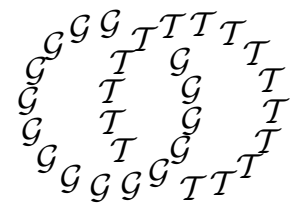

\title{
Toward a general theory of linking invariants
}

\author{
Vladimir V CHERNOV \\ YULI B RUdYAK \\ Department of Mathematics, 6188 Bradley Hall \\ Dartmouth College, Hanover NH 03755-3551, USA \\ and \\ Department of Mathematics, University of Florida \\ 358 Little Hall, Gainesville, FL 32611-8105, USA \\ Email: Vladimir.Chernov@dartmouth.edu and rudyak@math.ufl.edu
}

\begin{abstract}
Let $N_{1}, N_{2}, M$ be smooth manifolds with $\operatorname{dim} N_{1}+\operatorname{dim} N_{2}+1=\operatorname{dim} M$ and let $\phi_{i}$, for $i=1,2$, be smooth mappings of $N_{i}$ to $M$ where $\operatorname{Im} \phi_{1} \cap \operatorname{Im} \phi_{2}=\emptyset$. The classical linking number $\operatorname{lk}\left(\phi_{1}, \phi_{2}\right)$ is defined only when $\phi_{1 *}\left[N_{1}\right]=\phi_{2 *}\left[N_{2}\right]=0 \in H_{*}(M)$.

The affine linking invariant alk is a generalization of $\mathrm{lk}$ to the case where $\phi_{1 *}\left[N_{1}\right]$ or $\phi_{2 *}\left[N_{2}\right]$ are not zero-homologous. In [7] we constructed the first examples of affine linking invariants of nonzero-homologous spheres in the spherical tangent bundle of a manifold, and showed that alk is intimately related to the causality relation of wave fronts on manifolds. In this paper we develop the general theory.

The invariant alk appears to be a universal Vassiliev-Goussarov invariant of order $\leq 1$. In the case where $\phi_{1 *}\left[N_{1}\right]=\phi_{2 *}\left[N_{2}\right]=0 \in H_{*}(M)$, it is a splitting of the classical linking number into a collection of independent invariants.

To construct alk we introduce a new pairing $\mu$ on the bordism groups of spaces of mappings of $N_{1}$ and $N_{2}$ into $M$, not necessarily under the restriction $\operatorname{dim} N_{1}+\operatorname{dim} N_{2}+$ $1=\operatorname{dim} M$. For the zero-dimensional bordism groups, $\mu$ can be related to the HatcherQuinn invariant. In the case $N_{1}=N_{2}=S^{1}$, it is related to the Chas-Sullivan string homology super Lie bracket, and to the Goldman Lie bracket of free loops on surfaces.
\end{abstract}

AMS Classification numbers Primary: 57R19

Secondary: 14M07, 53Z05, 55N22, 55N45, 57M27, 57R40, 57R45, 57R52

Keywords: Linking invariants, winding numbers, Goldman bracket, wave fronts, causality, bordisms, intersections, isotopy, embeddings

Proposed: Steve Ferry

Seconded: Ralph Cohen, Leonid Polterovich
Received: 30 January 2004

Revised: 20 September 2005 


\section{Introduction}

In this paper the word "smooth" means $C^{\infty}$. Throughout this paper $M$ is a smooth connected oriented manifold (not necessarily compact), and $N_{1}, N_{2}$ are smooth oriented closed manifolds. The dimensions of $M, N_{1}, N_{2}$ are denoted by $m, n_{1}, n_{2}$, respectively, and the one-point space is denoted by $\mathrm{pt}$.

Let $\mathcal{N}_{i}$, for $i=1,2$, be a path-connected component of the space of all smooth mappings of $N_{i}$ to $M$. (Thus the mappings in $\mathcal{N}_{i}, i=1,2$, are not assumed to be immersions.) Let $\mathcal{B}=\mathcal{B}_{\mathcal{N}_{1}, \mathcal{N}_{2}}$ be the space of quadruples $\left(\phi_{1}, \phi_{2}, \rho_{1}, \rho_{2}\right)$ where $\phi_{i}: N_{i} \rightarrow M, i=1,2$, belong to $\mathcal{N}_{i}$ and $\rho_{i}:$ pt $\rightarrow N_{i}$ are such that $\phi_{1} \rho_{1}=\phi_{2} \rho_{2}$. Clearly, $\mathcal{B}$ can be regarded as a subset of $\mathcal{N}_{1} \times \mathcal{N}_{2} \times N_{1} \times N_{2}$, and we equip $\mathcal{B}$ with the subspace topology.

The classical linking number $\mathrm{lk}$ is a $\mathbb{Z}$-valued invariant of a pair $\left(\phi_{1}, \phi_{2}\right) \in$ $\mathcal{N}_{1} \times \mathcal{N}_{2}$ with $n_{1}+n_{2}+1=m$ (and with $\phi_{1}\left(N_{1}\right) \cap \phi_{2}\left(N_{2}\right)=\emptyset$ ). The invariant $\operatorname{lk}\left(\phi_{1}, \phi_{2}\right)$ is defined only if $\phi_{1 *}\left(\left[N_{1}\right]\right), \phi_{2 *}\left(\left[N_{2}\right]\right)=0 \in H_{*}(M)$ (or if $\phi_{1 *}\left(\left[N_{1}\right]\right), \phi_{2 *}\left(\left[N_{2}\right]\right)$ are torsion classes, in which case lk takes values in $\mathbb{Q}$ or $\mathbb{Q} / \mathbb{Z})$. U Kaiser [16] generalized linking numbers to the case of arbitrary submanifolds of the linking dimension that are homologous into a boundary or into an end of the ambient manifold. (For $M$ being the solid torus the similar approach to defining linking numbers was previously used by S Tabachnikov [30.)

The main goal of the paper is to construct a version of the linking invariant $\mathrm{lk}$ for pairs $\left(\phi_{1}, \phi_{2}\right) \in \mathcal{N}_{1} \times \mathcal{N}_{2}$ with $n_{1}+n_{2}+1=m$ and $\phi_{1}\left(N_{1}\right) \cap \phi_{2}\left(N_{2}\right)=\emptyset$ without any restrictions on the homology classes $\phi_{1 *}\left(\left[N_{1}\right]\right), \phi_{2 *}\left(\left[N_{2}\right]\right)$.

In greater detail, let $\Sigma \in \mathcal{N}_{1} \times \mathcal{N}_{2}$ be the subset of pairs $\left(\phi_{1}, \phi_{2}\right)$ with $\phi_{1}\left(N_{1}\right) \cap$ $\phi_{2}\left(N_{2}\right) \neq \emptyset$. Fixing a pair $* \in \mathcal{N}_{1} \times \mathcal{N}_{2} \backslash \Sigma$, we define an invariant

$$
\text { alk: } \mathcal{N}_{1} \times \mathcal{N}_{2} \backslash \Sigma \rightarrow H_{0}(\mathcal{B}) / \text { Indet }
$$

which is an invariant under link homotopy of pairs $\left(\phi_{1}, \phi_{2}\right)$; here Indet is a certain indeterminacy subgroup. We call alk the affine linking invariant, since the change of the base point $*$ leads to changing of alk by an additive constant.

It turns out that the augmentation $\mathcal{B} \rightarrow$ pt reduces our invariants to the classical ones (ie the linking numbers with values in $H_{0}(\mathrm{pt})=\mathbb{Z}$ ) provided that the last ones are defined. In other words, here we have a splitting of the classical linking invariant.

Our constructions can be easily modified to yield affine linking type invariants under the singular concordance relation. In the case of 1-links in 3-manifolds this will give us the invariants constructed by Schneiderman in [26]. 
Our construction can be rather easily modified to give an invariant of a pair $\left(\phi_{1}, \phi_{2}\right) \in \mathcal{N}_{1} \times \mathcal{N}_{2}$ with $\phi_{1}\left(N_{1}\right) \cap \phi_{2}\left(N_{2}\right)=\emptyset$ and without any restrictions on the dimensions $n_{1}, n_{2}, m$ of $N_{1}, N_{2}, M$. In this case we also get an invariant of a link $\left(\phi_{1}, \phi_{2}\right)$ considered up to the link homotopy. The invariant takes values in $\Omega_{n_{1}+n_{2}+1-m}(\mathcal{B}) /\left(\operatorname{Im} \mu_{1,0}+\operatorname{Im} \mu_{0,1}\right)$, where $\mu_{i, j}: \Omega_{i}\left(\mathcal{N}_{1}\right) \otimes \Omega_{j}\left(\mathcal{N}_{2}\right) \rightarrow$ $\Omega_{i+j+n_{1}+n_{2}-m}(\mathcal{B})$ is the pairing defined in Theorem 2.2. We plan to study this more general invariant in detail in another paper.

Most of our results are based on considering of a helpful pairing

$$
\mu_{i, j}: \Omega_{i}\left(\mathcal{N}_{1}\right) \otimes \Omega_{j}\left(\mathcal{N}_{2}\right) \rightarrow \Omega_{i+j+n_{1}+n_{2}-m}(\mathcal{B})
$$

where $\Omega_{*}(X)$ is the group of oriented bordisms of $X$. This pairing has many remarkable properties. For example:

(1) The pairings $\mu_{1,0}, \mu_{0,1}$ enable us to describe the indeterminacy for the invariant alk. (Note that $H_{0}(X)=\Omega_{0}(X)$ for all $X$.)

(2) The pairing $\mu_{0,0}$ tells us (in many cases) whether two $C^{\infty}$ maps $f_{1}: N_{1} \rightarrow$ $M$ and $f_{2}: N_{2} \rightarrow M$ can be deformed to maps with disjoint images, see Section 8. The case when two immersions $f_{1}$ and $f_{2}$ can be regularly homotoped to maps with disjoint images was considered by Hatcher and Quinn [15]. Concerning relations between $\mu_{0,0}$ and the Hatcher-Quinn invariant, see subsection 2.2. A conicidence problem for the case $N_{1}=N_{2}$ was considered by Koschorke [18] via the approach of Hatcher-Quinn invariants.

(3) If $N_{1}=N_{2}=S^{n}$ and $M$ is a $2 n$-manifold then $\mu$ leads to a generalization of the Goldman bracket [12] of free loops on 2-surfaces, see subsection 2.1

(4) In case of $N_{1}=N_{2}=S^{1}$ the pairing $\mu$ leads to a (graded) Lie algebra structure on $\Omega_{*}(\overline{\mathcal{N}})$ where $\overline{\mathcal{N}}=\overline{\mathcal{N}}_{1}=\overline{\mathcal{N}}_{2}$ is the union of all the connected components of the space of mappings $S^{1} \rightarrow M$. This Lie algebra structure is related to the string homology Lie bracket introduced by Chas and Sullivan [4, [5], cf also the work of A Voronov [36]. We are not able to discuss this algebra in detail here but intend to do it in the coming development of our work [9].

(5) In fact the mapping $\mu$ extends to a Lie bracket on the nonoriented bordism groups of mappings into $M$ of garlands glued out of arbitrary manifolds. It also seems that for the appropriately chosen signs $\mu$ extends to a (super) Lie bracket even for oriented bordism groups, but we are still computing the appropriate signs in the graded Jacobi identity, see [9, Theorem 3.1].

The paper is organized as follows. In Section 2 we introduce the pairing $\mu$. In Sections 3 and 4 we define affine linking invariants of pairs $\left(\phi_{1}, \phi_{2}\right) \in \mathcal{N}_{1} \times \mathcal{N}_{2}$ 
(with $f_{1}\left(N_{1}\right) \cap f_{2}\left(N_{2}\right)=\emptyset$ ) as elements of the group $\Omega_{0}(\mathcal{B})$ modulo certain indeterminacy; the last one is described in terms of the pairing $\mu$. In Section 5 we prove that the augmentation $\Omega_{0}(\mathcal{B})=H_{0}(\mathcal{B}) \rightarrow H_{0}(\mathrm{pt})=\mathbb{Z}$ induced by the mapping $\mathcal{B} \rightarrow$ pt reduces our invariants to the classical linking invariant, when the last one is well-defined. In Section [ 6 we give conditions that guarantee the vanishing of the indeterminacy. In Section 7 we give an explicit description of $\pi_{0}(\mathcal{B})$ and $\Omega_{0}(\mathcal{B})$. In Section 8 we show that, in many cases, the pair $\bar{f}_{1}: N_{1} \rightarrow M, \bar{f}_{2}: N_{2} \rightarrow M$ of maps, with $n_{1}+n_{2}=m$ is homotopic to a pair $\left(f_{1}, f_{2}\right)$ with disjoint images, $f_{1}\left(N_{1}\right) \cap f_{2}\left(N_{2}\right)=\emptyset$, if and only if the pairing $\mu$ takes the zero value on $\left(\bar{f}_{1}, \bar{f}_{2}\right)$. If $\bar{f}_{1}$ and $\bar{f}_{2}$ are homotopic to immersions, then the results of Section 8 follow immediately from Theorem 2.2 of HatcherQuinn [15].

Acknowledgements The first author was partially supported by the Walter and Constance Burke Research Initiation Award. The second author was partially supported by NSF, grant 0406311, and by MCyT, projects BFM 200200788 and MCyT BFM2003-02068/MATE, Spain; his visit to Dartmouth College was supported by the funds donated by Edward Shapiro to the Mathematics Department of Dartmouth College.

We are also grateful to the anonymous referee for the very valuable comments and suggestions.

\section{$2 \quad$ The pairing $\mu_{i, j}: \Omega_{i}\left(\mathcal{N}_{1}\right) \otimes \Omega_{j}\left(\mathcal{N}_{2}\right) \rightarrow \Omega_{i+j+n_{1}+n_{2}-m}(\mathcal{B})$}

In this section we do not assume that $\operatorname{dim} N_{1}+\operatorname{dim} N_{2}+1=\operatorname{dim} M$.

Given a space $X$, we denote by $\Omega_{n}(X)$ the $n$-dimensional oriented bordism group of $X$. Recall that $\Omega_{n}(X)$ is the set of the equivalence classes of (continuous) maps $f: V^{n} \rightarrow X$ where $V$ is a closed oriented manifold. Here two maps $f_{1}: V_{1} \rightarrow X$ and $f_{2}: V_{2} \rightarrow X$ are equivalent if there exists a map $g: W^{n+1} \rightarrow X$, where $W$ is a compact oriented manifold whose oriented boundary $\partial W$ is diffeomorphic to $V_{1} \sqcup\left(-V_{2}\right)$ and $\left.g\right|_{\partial W}=f_{1} \sqcup f_{2}$. Furthermore, the operation of disjoint union converts $\Omega_{n}(X)$ into an abelian group. See 25, 28, 29] for details.

Let $[V] \in H_{n}(V)$ be the fundamental class of a closed oriented $n$-dimensional manifold $V$. Every map $f: V \rightarrow X$ gives us an element $f_{*}[V] \in H_{n}(X)$, and the correspondence $(V, f) \mapsto f_{*}[V]$ yields the Steenrod-Thom homomorphism

$$
\tau: \Omega_{n}(X) \rightarrow H_{n}(X)
$$


It turns out 31] that this homomorphism is an isomorphism for $n \leq 3$ and an epimorphism for $n \leq 6$, see [25, 28] for modern proofs.

Let $\alpha_{1}: F_{1} \rightarrow \mathcal{N}_{1}$ be a mapping representing $\left[\alpha_{1}\right] \in \Omega_{i}\left(\mathcal{N}_{1}\right)$ and let $\alpha_{2}: F_{2} \rightarrow$ $\mathcal{N}_{2}$ be a mapping representing $\left[\alpha_{2}\right] \in \Omega_{j}\left(\mathcal{N}_{2}\right)$. Let $\widetilde{\alpha}_{i}: F_{i} \times N_{i} \rightarrow M, i=1,2$, be such that $\widetilde{\alpha}_{i}(f, n)=\left(\alpha_{i}(f)\right)(n)$. Let $v_{1} \in F_{1} \times N_{1}$ and $v_{2} \in F_{2} \times N_{2}$ be such that $\widetilde{\alpha}_{1}\left(v_{1}\right)=\widetilde{\alpha}_{2}\left(v_{2}\right)$. We say that $\widetilde{\alpha}_{1}$ and $\widetilde{\alpha}_{2}$ are transverse at $\left(v_{1}, v_{2}\right)$ if $d \widetilde{\alpha}_{2}\left(T_{v_{2}}\left(F_{2} \times N_{2}\right)\right)$ and $d \widetilde{\alpha}_{1}\left(T_{v_{1}}\left(F_{1} \times N_{1}\right)\right)$ generate $T_{\widetilde{\alpha}_{1}\left(v_{1}\right)} M=T_{\widetilde{\alpha}_{2}\left(v_{2}\right)} M$. Following standard arguments we can assume that $\widetilde{\alpha}_{1}$ and $\widetilde{\alpha}_{2}$ are transverse, ie they are transverse at all $\left(v_{1}, v_{2}\right)$ such that $\widetilde{\alpha}_{1}\left(v_{1}\right)=\widetilde{\alpha}_{2}\left(v_{2}\right)$.

Consider the pullback diagram

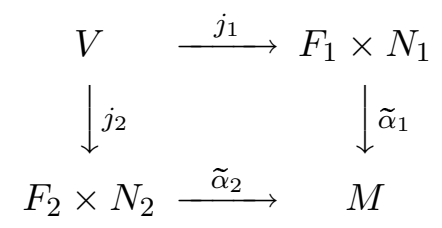

of the maps $\widetilde{\alpha}_{i}$, for $i=1,2$.

Lemma 2.1 If $\widetilde{\alpha}_{1}, \widetilde{\alpha}_{2}$ are transverse, then $V$ is a closed oriented $(i+j+$ $\left.n_{1}+n_{2}-m\right)$-dimensional manifold.

Let $p_{1}: F_{i} \times N_{i} \rightarrow F_{i}$ and $p_{2}: F_{i} \times N_{i} \rightarrow N_{i}$, for $i=1,2$, be the obvious projections. Consider the mapping

$$
\mu\left(\widetilde{\alpha}_{1}, \widetilde{\alpha}_{2}\right): V \rightarrow \mathcal{B}, \quad v \mapsto\left(\phi_{1}^{v}, \phi_{2}^{v}, \rho_{1}^{v}, \rho_{2}^{v}\right)
$$

where $\phi_{i}^{v}(n)=\widetilde{\alpha}_{i}\left(p_{1}\left(j_{i}(v)\right), n\right)$ for $n \in N_{i}$, and $\rho_{i}^{v}(\mathrm{pt})=p_{2}\left(j_{i}(v)\right)$.

Theorem 2.2 The above construction yields a well-defined pairing

$$
\begin{aligned}
\mu=\mu_{i j}: \Omega_{i}\left(\mathcal{N}_{1}\right) \otimes \Omega_{j}\left(\mathcal{N}_{2}\right) & \rightarrow \Omega_{i+j+n_{1}+n_{2}-m}(\mathcal{B}), \\
\mu\left(\left[\alpha_{1}\right],\left[\alpha_{2}\right]\right) & =\left[V, \mu\left(\widetilde{\alpha}_{1}, \widetilde{\alpha}_{2}\right)\right] .
\end{aligned}
$$

Proof The routine argument shows that the bordism class $\left[V, \mu\left(\widetilde{\alpha}_{1}, \widetilde{\alpha}_{2}\right)\right]$ in $\Omega_{i+j+n_{1}+n_{2}-m}(\mathcal{B})$ depends only on $\left[\alpha_{1}\right] \in \Omega_{i}\left(\mathcal{N}_{1}\right),\left[\alpha_{2}\right] \in \Omega_{j}\left(\mathcal{N}_{2}\right)$. The bilinearity of $\mu$ is obvious. 


\subsection{Relation to the Goldman bracket}

Let $\widetilde{\mathcal{N}}_{1}$ (respectively, $\widetilde{\mathcal{N}}_{2}$ ) be the topological space that is the union of all the connected components of the space of mappings of $N_{1}$ (respectively, $N_{2}$ ) into $M$. Let $\widetilde{\mathcal{B}}$ be the topological space that is the union of $\mathcal{B}_{\mathcal{N}_{1}, \mathcal{N}_{2}}$ over all the connected components $\mathcal{N}_{1}, \mathcal{N}_{2}$.

Let $\overline{\mathcal{N}}_{i}$ be the closure of $\widetilde{\mathcal{N}}_{i}$ in the space of all continuous maps. Similarly, define $\overline{\mathcal{B}}$ to be the closure of $\widetilde{\mathcal{B}}$.

Clearly, the pairing $\mu$ can be extended to

$$
\bar{\mu}: \Omega_{i}\left(\overline{\mathcal{N}}_{1}\right) \otimes \Omega_{j}\left(\overline{\mathcal{N}}_{2}\right) \rightarrow \Omega_{i+j+n_{1}+n_{2}-m}(\overline{\mathcal{B}}) .
$$

Let $M$ be a $2 n$-dimensional manifold and let $N_{1}=N_{2}=S^{n}$. Given two points $x, y \in S^{n}$ (not necessarily distinct), choose an orientation preserving diffeomorphism $u_{x, y}: S^{n} \rightarrow S^{n}$ that maps $x$ to $y$.

For $M^{2 n}$ and $N_{1}=N_{2}=S^{n}$ put $\overline{\mathcal{N}}=\overline{\mathcal{N}}_{1}=\overline{\mathcal{N}}_{2}$ and consider the pairing

$$
\bar{\mu}: \Omega_{0}(\overline{\mathcal{N}}) \otimes \Omega_{0}(\overline{\mathcal{N}}) \rightarrow \Omega_{0}(\overline{\mathcal{B}}) .
$$

Since $\Omega_{0}=H_{0}$, the pairing (2.2) yields a pairing

$$
H_{0}(\overline{\mathcal{N}}) \otimes H_{0}(\overline{\mathcal{N}}) \stackrel{\bar{\mu}}{\longrightarrow} H_{0}(\overline{\mathcal{B}}) .
$$

Every point $b=\left(\phi_{1}, \phi_{2}, \rho_{1}, \rho_{2}\right) \in \overline{\mathcal{B}}$ gives us a map $h_{b}: S^{n} \vee S^{n} \rightarrow M$ as follows. We regard the sphere $S^{n}$ as a pointed space with the base point $*$. Clearly, both maps $\phi_{i} u_{*, \rho_{i}(\mathrm{pt})}: S^{n} \rightarrow M$, for $i=1,2$, map the point $*$ to the same point of $M$ and therefore yield the map $S^{n} \vee S^{n} \rightarrow M$.

The pairing $\mu: H_{0}(\overline{\mathcal{N}}) \otimes H_{0}(\overline{\mathcal{N}}) \rightarrow H_{0}(\overline{\mathcal{B}})$ has the following interpretation. Put $\widehat{\pi}_{n}$ to be the orbits of $\pi_{n}(M)$ under the natural action of $\pi_{1}(M)$. Then $H_{0}(\overline{\mathcal{N}})$ is naturally identified with $\mathbb{Z} \widehat{\pi}_{n}$, a free $\mathbb{Z}$-module over $\widehat{\pi}_{n}$. Observe that any two modules $\mathbb{Z} \widehat{\pi}_{n}(M, p)$ and $\mathbb{Z} \widehat{\pi}_{n}(M, q)$ are canonically isomorphic

Let $s_{i}: S^{n} \rightarrow M^{2 n}$, for $i=1,2$, be two smooth generic mappings transverse to each other and realizing $\left[s_{i}\right] \in \widehat{\pi}_{n}$. Here genericity means that each intersection point of $s_{1}$ and $s_{2}$ is not a self-intersection point of $s_{1}$ or $s_{2}$. Put $P=\operatorname{Im}\left(s_{1}\right) \cap$ $\operatorname{Im}\left(s_{2}\right)$. Then

$$
\bar{\mu}\left(1\left[s_{1}\right] \otimes 1\left[s_{2}\right]\right)=\sum_{p \in P} \operatorname{sign}(p)\left[s_{1}, s_{2}, p\right],
$$

where $\operatorname{sign} p$ is the natural orientation of $p$ coming from the intersection pairing. Here $\left[s_{1}, s_{2}, p\right] \in \pi_{0}(\overline{\mathcal{B}})$ is the element that maps the first sphere as $s_{1}$, the second sphere as $s_{2}$, and maps pt to the preimages of $p$ on the two spheres. 
Now, using the coproduct $S^{n} \rightarrow S^{n} \vee S^{n}$, we conclude that every $b \in \overline{\mathcal{B}}$ gives us a map

$$
S^{n} \longrightarrow S^{n} \vee S^{n} \stackrel{h_{b}}{\longrightarrow} M
$$

So, we get a map $\varphi: \overline{\mathcal{B}} \rightarrow \overline{\mathcal{N}}$. Notice that this map is not continuous, but it induces a well-defined map $\pi_{0}(\overline{\mathcal{B}}) \rightarrow \pi_{0}(\overline{\mathcal{N}})$ since each $u_{x, y}$ is homotopic to the identity. Furthermore, since $\Omega_{0}=H_{0}$, the pairing (2.2) yields a pairing

$$
\alpha: H_{0}(\overline{\mathcal{N}}) \otimes H_{0}(\overline{\mathcal{N}}) \stackrel{\bar{\mu}}{\longrightarrow} H_{0}(\overline{\mathcal{B}}) \stackrel{\varphi_{*}}{\longrightarrow} H_{0}(\overline{\mathcal{N}}) .
$$

Now, because of the equality (2.3), we conclude that

$$
\alpha\left(1\left[s_{1}\right] \otimes 1\left[s_{2}\right]\right)=\left(\sum_{p \in P} \operatorname{sign}(p)\left[s_{1} s_{2} \in \pi_{n}(M, p)\right]\right) \in \mathbb{Z} \widehat{\pi}_{n} .
$$

Here the element $\left[s_{1} s_{2} \in \pi_{n}(M, p)\right]$ is the class in $\widehat{\pi}_{n}$ that is the product of the elements of $\pi_{n}(M, p)$ realized by $s_{1}$ and $s_{2}$. More accurately, we have to compose $s_{i}$ with an automorphism $u_{*, x_{i}}$ of $S^{n}$ that maps the base point $*$ to the preimage $x_{i}=s_{i}^{-1}(p)$ of $p$.

For $n=1$ the action of $\pi_{1}(M)$ on $\pi_{n}(M)=\pi_{1}(M)$ is given by the conjugation. So $\mathbb{Z} \widehat{\pi}_{n}=\mathbb{Z} \widehat{\pi}_{1}$ is a free $\mathbb{Z}$-module generated by the homotopy classes of free loops on $M$. Because of the explanations above $\left[s_{1} s_{2} \in \pi_{n}(M, p)\right]$ is a welldefined element of $\mathbb{Z} \widehat{\pi}_{1}$.

Now formula (2.4) is identical to the definition of Goldman's Lie bracket on $\mathbb{Z} \widehat{\pi}_{1}\left(M^{2}\right)$, see Goldman [12, page 267]. Since both $\alpha$ and Goldman's Lie bracket are bilinear and coincide on the generators of $\mathbb{Z} \widehat{\pi}_{1}$, they are equal. Thus for $N_{1}=N_{2}=S^{1}$ and $M^{2}$ our pairing $\mu$ generalizes Goldman's Lie bracket discovered in stages by Goldman [12] and Turaev [32].

\subsection{Relation to the Hatcher-Quinn invariant}

Consider two maps $f: N_{1} \rightarrow M$ and $g: N_{2} \rightarrow M$ in $\mathcal{N}_{1}$ and $\mathcal{N}_{2}$, respectively. Hatcher and Quinn [15] considered the homotopy pullback diagram

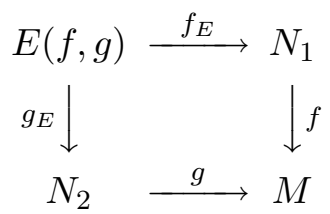

For $i=1,2$, let $\nu_{i}$ be the stable normal vector bundle over $N_{i}$ and let $\xi$ be the stable vector bundle $f_{E}^{*} \nu_{1} \oplus g_{E}^{*} \nu_{2} \oplus f_{E}^{*} f^{*} \tau_{M}$. Let $E$ denote $E(f, g)$ and let 
$\Omega_{k}^{\mathrm{fr}}(E, \xi)$ denote the bordism group based on singular manifolds $h: V^{k} \rightarrow E$ together with a stable bundle isomorphism of the normal bundle of $V$ with $h^{*} \xi$. The $\Omega_{k}^{\mathrm{fr}}(E, \xi)$ groups are the homotopy groups of the Thom spectrum $T \xi$.

Given two transverse maps $f_{1}: N_{1} \rightarrow M$ and $f_{2}: N_{2} \rightarrow M$ homotopic to $f$ and $g$ respectively, consider the pullback $V$ (rather than the homotopy pullback) of $f_{1}$ and $f_{2}$. Consider the obvious maps $v_{i}: V \rightarrow N_{i}$ and construct a map $h: V \rightarrow E$ with $f_{E} \circ h=v_{1}$ and $g_{E} \circ h=v_{2}$. In fact, this $h$ determines and is determined by homotopies from $f_{1}$ to $f$ and from $f_{2}$ to $g$. Then $(V, h)$ yields an element of $\Omega_{n_{1}+n_{2}-m}^{\mathrm{fr}}(E, \xi)$, and this element does not depend on the choice of the above described homotopies. So, we have the Hatcher-Quinn map

$$
\text { hq: } \pi_{0}\left(\mathcal{N}_{1}\right) \times \pi_{0}\left(\mathcal{N}_{2}\right) \longrightarrow \Omega_{n_{1}+n_{2}-m}^{\mathrm{fr}}(E, \xi) .
$$

Now, assume that $n_{1}+n_{2}=m$. Take a point of $\mathcal{B}$ and represent it by a commutative diagram

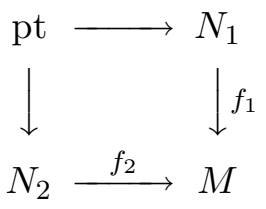

with $f_{1}, f_{2}$ transverse and $f_{1} \simeq f, f_{2} \simeq g$. Clearly, this diagram gives us an element of the group $\Omega_{0}^{\mathrm{fr}}(E, \xi)$, and in fact we have a map $\varphi: \Omega_{0}(\mathcal{B}) \rightarrow$ $\Omega_{0}^{\mathrm{fr}}(E, \xi)$. It is easy to see that the diagram

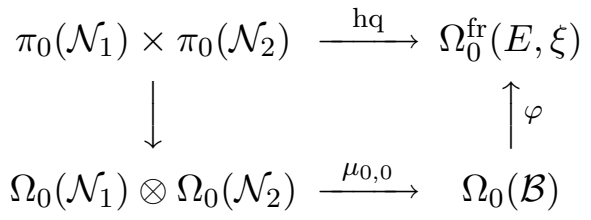

commutes. So in the case $n_{1}+n_{2}=m$ the pairing $\mu_{0,0}$ can be regarded as a version of the Hatcher-Quinn invariant.

\section{Affine linking invariants}

From here and till the end of the paper we assume that $n_{1}+n_{2}+1=m$ (unless the opposite is explicitly stated).

Put $\Sigma$ to be the discriminant in $\mathcal{N}_{1} \times \mathcal{N}_{2}$, ie the subspace that consists of pairs $\left(f_{1}, f_{2}\right)$ such that there exist $y_{1} \in N_{1}, y_{2} \in N_{2}$ with $f_{1}\left(y_{1}\right)=f_{2}\left(y_{2}\right)$. (We do not include into $\Sigma$ the maps that are singular in the common sense but do not involve double points between $f_{1}\left(N_{1}\right)$ and $f_{2}\left(N_{2}\right)$.) 
Definition 3.1 We define $\Sigma_{0}$ to be the subset (stratum) of $\Sigma$ consisting of all the pairs $\left(f_{1}, f_{2}\right)$ such that there exists precisely one pair $\left(y_{1}, y_{2}\right)$ of points $y_{1} \in N_{1}, y_{2} \in N_{2}$ such that

(a) $f_{1}\left(y_{1}\right)=f_{2}\left(y_{2}\right)$;

(b) $y_{i}$ is a regular point of $f_{i}$, for $i=1,2$;

(c) $\left(d f_{1}\right)\left(T_{y_{1}} N_{1}\right) \cap\left(d f_{2}\right)\left(T_{y_{2}} N_{2}\right)=0$.

Construction 3.2 Let $\gamma:(a, b) \rightarrow \mathcal{N}_{1} \times \mathcal{N}_{2}$ be a path which intersects $\Sigma_{0}$ in a point $\gamma\left(t_{0}\right)$. We also assume that

$$
\gamma\left(t_{0}-\delta, t_{0}+\delta\right) \cap \Sigma=\gamma\left(t_{0}\right)
$$

for $\delta$ small enough. We construct a vector $\mathbf{v}=\mathbf{v}\left(\gamma, t_{0}, \delta\right)$ as follows. We regard $\gamma\left(t_{0}\right)$ as a pair $\left(f_{1}, f_{2}\right) \in \mathcal{N}_{1} \times \mathcal{N}_{2}$ and consider the points $y_{1}, y_{2}$ as in Definition 3.1. Set $z=f_{1}\left(y_{1}\right)=f_{2}\left(y_{2}\right)$. Choose a small $\delta>0$ and regard $\gamma\left(t_{0}+\delta\right)$ as a pair $\left(g_{1}, g_{2}\right) \in \mathcal{N}_{1} \times \mathcal{N}_{2}$. Set $z_{i}=g_{i}\left(y_{i}\right), i=1,2$. Take a chart for $M$ that contains $z$ and $z_{i}, i=1,2$, and set

$$
\mathbf{v}\left(\gamma, t_{0}, \delta\right):=\overrightarrow{z z_{1}}-\overrightarrow{z z_{2}} \in T_{z} M
$$

Definition 3.3 Let $\gamma:(a, b) \rightarrow \mathcal{N}_{1} \times \mathcal{N}_{2}$ be a path as in Construction 3.2 We say that $\gamma$ intersects $\Sigma_{0}$ transversally for $t=t_{0}$ if there exists $\delta_{0}>0$ such that

$$
\mathbf{v}\left(\gamma, t_{0}, \delta\right) \notin\left(d f_{1}\right)\left(T_{y_{1}} N_{1}\right) \oplus\left(d f_{2}\right)\left(T_{y_{2}} N_{2}\right) \subset T_{z} M
$$

for all $\delta \in\left(0, \delta_{0}\right)$.

It is easy to see that the concept of transverse intersection does not depend on the choice of the chart.

Definition 3.4 A path $\gamma:(a, b) \rightarrow \mathcal{N}_{1} \times \mathcal{N}_{2},-\infty \leq a<b \leq \infty$ is said to be generic if

(a) $\gamma(a, b) \cap \Sigma=\gamma(a, b) \cap \Sigma_{0}$;

(b) the set $J=\left\{t \mid \gamma(t) \cap \Sigma_{0} \neq \emptyset\right\} \subset(a, b)$ is an isolated subset of $\mathbb{R}$;

(c) the path $\gamma$ intersects $\Sigma_{0}$ transversally for all $t \in J$.

As one can expect, every path can be turned into a generic one by a small deformation. We leave the proof to the reader. 
Definition 3.5 Let $\gamma$ be a path in $\mathcal{N}_{1} \times \mathcal{N}_{2}$ that intersects $\Sigma$ transversally in one point $\gamma\left(t_{0}\right) \in \Sigma_{0}$. We associate a sign $\sigma\left(\gamma, t_{0}\right)$ to such a crossing as follows.

We regard $\gamma\left(t_{0}\right)$ as a pair $\left(f_{1}, f_{2}\right) \in \mathcal{N}_{1} \times \mathcal{N}_{2}$ and consider the points $y_{1} \in$ $N_{1}, y_{2} \in N_{2}$ such that $f_{1}\left(y_{1}\right)=f_{2}\left(y_{2}\right)$. Set $z=f_{1}\left(y_{1}\right)=f_{2}\left(y_{2}\right)$. Let $\mathfrak{r}_{1}$ and $\mathfrak{r}_{2}$ be frames which are tangent to $N_{1}$ and $N_{2}$ at $y_{1}$ and $y_{2}$, respectively, and both are assumed to be positive. Consider the frame

$$
\left\{d f_{1}\left(\mathfrak{r}_{1}\right), \mathbf{v}, d f_{2}\left(\mathfrak{r}_{2}\right)\right\}
$$

at $z \in M$, where $\mathbf{v}$ is a vector described in Construction 3.2. We put $\sigma\left(\gamma, t_{0}\right)=$ 1 if this frame gives us the orientation of $M$, otherwise we put $\sigma\left(\gamma, t_{0}\right)=-1$. Because of the transversality and condition (c) from Definition 3.1] the family $\left\{d f_{1}\left(\mathfrak{r}_{1}\right), \mathbf{v}, d f_{2}\left(\mathfrak{r}_{2}\right)\right\}$ is indeed a frame. Note also that the vector $\mathbf{v}$ is not welldefined, but the above defined sign $\sigma$ is.

Clearly if we traverse the path $\gamma$ in the opposite direction then the sign of the crossing changes.

For every space $X$, the group $\Omega_{0}(X)=H_{0}(X)$ is the free abelian group with the base $\pi_{0}(X)$. So, every element of $\Omega_{0}(X)$ can be represented as a finite linear combination $\sum \lambda_{k} P_{k}$ with $\lambda_{k} \in \mathbb{Z}$ and $P_{k} \in X$, and every such linear combination gives us an element of $\Omega_{0}(X)$. Below in Section 7 we give examples of situations where $\pi_{0}(\mathcal{B})$ is an infinite set, in spite of the fact that $\mathcal{N}_{1}$ and $\mathcal{N}_{2}$ are path connected.

Definition 3.6 Let $\gamma$ be a path in $\mathcal{N}_{1} \times \mathcal{N}_{2}$ that intersects $\Sigma$ transversally in one point $\gamma\left(t_{0}\right) \in \Sigma_{0}$. We define $\left[\gamma\left(t_{0}\right)\right] \in \Omega_{0}(\mathcal{B})$ as $\sigma\left(t_{0}\right) \gamma\left(t_{0}\right)$.

Clearly,

$$
\varepsilon\left(\left[\gamma\left(t_{0}\right]\right)=\sigma\left(\gamma, t_{0}\right),\right.
$$

where $\varepsilon: \Omega_{0}(\mathcal{B}) \rightarrow \mathbb{Z}=\Omega_{0}(\mathrm{pt})$ is the homomorphism induced by the map $\mathcal{B} \rightarrow$ pt.

Definition 3.7 Since $\Omega_{i}(X)=H_{i}(X)$ for $i=0,1$, by the Künneth formula we have the canonical isomorphism

$$
\Omega_{1}(X \times Y)=\Omega_{1}(X) \otimes \Omega_{0}(Y) \oplus \Omega_{0}(X) \otimes \Omega_{1}(Y) .
$$

Now, the pairings

$$
\mu_{10}: \Omega_{1}\left(\mathcal{N}_{1}\right) \otimes \Omega_{0}\left(\mathcal{N}_{2}\right) \rightarrow \Omega_{0}(\mathcal{B}) \text { and } \mu_{01}: \Omega_{0}\left(\mathcal{N}_{1}\right) \otimes \Omega_{1}\left(\mathcal{N}_{2}\right) \rightarrow \Omega_{0}(\mathcal{B})
$$

yield the homomorphism

$$
\lambda: \Omega_{1}\left(\mathcal{N}_{1} \times \mathcal{N}_{2}\right) \rightarrow \Omega_{0}(\mathcal{B}) .
$$


We define the indeterminacy subgroup Indet $\subset \Omega_{0}(\mathcal{B})$ to be the image of the homomorphism $\lambda$. We define the abelian group $\mathbb{A}=\mathbb{A}\left(\mathcal{N}_{1}, \mathcal{N}_{2}\right)$ to be the quotient group of $\Omega_{0}(\mathcal{B}) /$ Indet. Let $q=q_{\mathcal{N}_{1}, \mathcal{N}_{2}}: \Omega_{0}(\mathcal{B}) \rightarrow \mathbb{A}$ be the corresponding quotient homomorphism.

Examples 3.8 (1) Consider the case of $M^{3}$ being a lens space with a fundamental group $\mathbb{Z}_{p}, N_{1}=S^{2}, N_{2}=$ pt. In this case there is only one homotopy class of mappings $S^{2} \rightarrow M$ and of pt $\rightarrow M$. It is easy to see that $\Omega_{0}(\mathcal{B})=\mathbb{Z}$.

Take $f: S^{2} \rightarrow M$ and $g:$ pt $\times S^{1}=S^{1} \rightarrow M$. Then $\mu_{0,1}(f, g)$ equals to the intersection index between $f_{*}\left[S^{2}\right]$ and $g_{*}\left[S^{1}\right] \in H_{*}(M)$. Since $H_{1}(M)=\mathbb{Z}_{p}$ we get that $\operatorname{Im} \mu_{0,1}=0 \in \mathbb{Z}=\Omega_{0}(\mathcal{B})$.

Take $r: S^{2} \times S^{1} \rightarrow M$ and $s:$ pt $\rightarrow M$. Then $\mu_{1,0}(r, s) \in \mathbb{Z}$ equals to the degree of the mapping $r: S^{2} \times S^{1} \rightarrow M$. Since $\pi_{2}(M)=0$, the elementary obstruction theory shows that for a given image of $r_{*}\left(\cdot \times S^{1}\right) \in \pi_{1}(M)$ all the homotopy classes of mappings $S^{2} \times S^{1} \rightarrow M$ are classified by $\pi_{3}(M)$. Thus $\operatorname{Im} \mu_{1,0}$ coincides with the possible degrees of mappings $S^{3} \rightarrow M$. Since all such mappings pass through the covering map $S^{3} \rightarrow M$ that has degree $p$, we get $\operatorname{Im} \mu_{1,0}=p \mathbb{Z} \subset \mathbb{Z}$. Thus Indet $=p \mathbb{Z} \subset \mathbb{Z}=\Omega_{0}(\mathcal{B})$.

(2) A harder example comes from $M=F_{g} \times S^{1}$, where $F_{g}$ is an oriented surface of genus $g>1$. Let $N_{1}=N_{2}=S^{1}$ and let $\alpha, \beta: S^{1} \rightarrow M$ be linked embedded circles that project to two simple curves on $F_{g}$ with one intersection point. Let $\mathcal{N}_{1}, \mathcal{N}_{2}$ be the connected components of the space of mappings $S^{1} \rightarrow M$ that contain $\alpha$ and $\beta$, respectively. Let $r:\left(S^{1}, *\right) \rightarrow\left(\mathcal{N}_{1}, \alpha\right)$ be a mapping with the adjoint $\hat{r}: S^{1} \times S^{1} \rightarrow M, \hat{r}_{\left.\right|^{1} \times 1}=\alpha$.

If $\operatorname{ker}\left(\hat{r}_{*}: \pi_{1}\left(S^{1} \times S^{1}\right) \rightarrow \pi_{1}(M)\right) \neq 1$, then using obstruction theory we get that $\hat{r}$ is homotopic to a mapping that passes through a mapping $S^{1} \rightarrow M$. This mapping of $S^{1}$ can be made disjoint from $\beta$ and $\mu_{1,0}(r, \beta)=0$ for such $r$.

If $\hat{r}_{*}$ is injective then $r$ is homotopic to the loop $\gamma_{1}^{i} \gamma_{3}^{j} \in\left(\mathcal{N}_{1}, \alpha\right)$, for some $i, j \in \mathbb{Z}$. Here $\gamma_{1}$ is a self homotopy of $\alpha$ induced by one full rotation of the parameterizing circle and $\gamma_{3}$ is a self homotopy of $\alpha$ under which every point of $\alpha$ slides one full turn along the $S^{1}$ fiber of $F_{g} \times S^{1} \rightarrow F_{g}$ that contains the point, see the proof of [6. Lemma 6.11]. Clearly $\mu_{1,0}\left(\gamma_{1}, \beta\right)=0$ and $\mu_{1,0}\left(\gamma_{3}, \beta\right)$ equals to a \pm 1 times the class of the element of $\mathcal{B}$ that is obtained when $\alpha$ intersects $\beta$ under the deformation $\gamma_{3}$. Thus $\operatorname{Im} \mu_{1,0}=\mathbb{Z} \subset \Omega_{0}(\mathcal{B})$.

Similarly one shows that $\operatorname{Im} \mu_{0,1}=\mathbb{Z} \subset \Omega_{0}(\mathcal{B})$ and that in fact $\operatorname{Im} \mu_{1,0}=$ $\operatorname{Im} \mu_{0,1}$. Thus Indet $=\mathbb{Z}$ for this example. One can also show that $\Omega_{0}(\mathcal{B})=$ $\bigoplus_{1}^{\infty} \mathbb{Z}$ in this case.

Similar construction for $F_{g}=S^{1} \times S^{1}$ will give Indet $=\mathbb{Z}=\Omega_{0}(\mathcal{B})$. 
Theorem-Definition 3.9 Let $\mathbb{A}$ be as above. Then there exists a function alk: $\mathcal{N}_{1} \times \mathcal{N}_{2} \backslash \Sigma \rightarrow \mathbb{A}$ such that

(a) alk is constant on path connected components of $\mathcal{N}_{1} \times \mathcal{N}_{2} \backslash \Sigma$;

(b) if $\gamma:[a, b] \rightarrow \mathcal{N}_{1} \times \mathcal{N}_{2}$ is a generic path such that $\gamma(a), \gamma(b) \notin \Sigma$ and $t_{i}, i \in I$, are the moments when $\gamma\left(t_{i}\right) \in \Sigma$ (and hence $\gamma\left(t_{i}\right) \in \Sigma_{0}$ by the definition of the generic path), then

$$
\operatorname{alk}(\gamma(b))-\operatorname{alk}(\gamma(a))=q\left(\sum_{i \in I}\left[\gamma\left(t_{i}\right)\right]\right) \in \mathbb{A} .
$$

Moreover, these properties determine the function alk uniquely up to an additive constant. We call such a function an affine linking invariant.

We prove Theorem 3.9 in Section 4

Remark 3.10 After the first version [10] of this text appeared on the electronic archive, U Koschorke submitted the paper [19] where he used some invariants coming from the Hatcher-Quinn construction to study when two submanifolds of $M=S \times \mathbb{R}$ can be link homotoped to the disjoint $S$-levels.

Remark 3.11 It is rather easy to prove (see Corollary [7.5) that the mapping alk: $\pi_{0}\left(\mathcal{N}_{1} \times \mathcal{N}_{2} \backslash \Sigma\right) \rightarrow \mathbb{A}$ is always surjective.

\subsection{Relations to front propagation and winding numbers}

(a) Let $S T M$ denote the total space of the sphere tangent bundle over $M$, $\operatorname{dim} S T M=2 m-1$. In [7] we defined the affine linking invariant al for the mappings of $S^{m-1} \rightarrow S T M$ that are homotopic to the inclusion of the fiber $S^{m-1}$ to $S T M$. Because of the orientability of the bundle $S T M \rightarrow M$, the homotopy class of this inclusion is invariant under the $\pi_{1}(M)$-action on $\left[S^{m-1}, S T M\right]$. Using Theorem 7.4 we get that in this case $\Omega_{0}(\mathcal{B})=\mathbb{Z}$, and al is exactly alk for $N_{1}=N_{2}=S^{m-1}$ and the space $\mathcal{N}_{1}=\mathcal{N}_{2}$ consisting of mappings $S^{m-1} \rightarrow S T M$ as above.

In 7] we have shown that in this case $\varepsilon$ (Indet $)=$ Indet $=0$ when $m$ is even or when $m$ is odd and $M$ is not a rational homology sphere. This shows that $\varepsilon($ alk $)$ can indeed be $\mathbb{Z}$-valued in many cases where the mappings are not zero-homologous.

This example is interesting since it is related to wave front propagation. Deep relations between front propagation and link theory were first discovered by 
V Arnold [2], who observed that the isotopy type of the knot canonically associated to a front does not change under front propagation. A wave front on $M$ is a singular spherical hypersurface equipped with a velocity vector field of the directions of the front propagation. The wave front represents the set of all points on $M$ that a certain information has reached at the given moment. Wave fronts on $M$ can be canonically lifted to $S T M$ by mapping a point of the front to the point of $S T M$ corresponding to the front velocity at this point. We assume that wave front propagation on $M$ is given by a time-dependent flow on STM. (For example light propagation is indeed given by such a flow.)

Consider two wave fronts that originated at two different points on $M$. The value of the alk invariant on the canonical lifts to $S T M$ of the two wave fronts equals to the algebraic number of times the front of the earlier event has passed through the birth point of the later event before the later event occurred, see [7]. Thus if the alk invariant is nonzero we conclude that the later event occurred after the earlier born front passed through its birth point. Philosophically this means that the later event has obtained the information about the earlier event carried by its wave front. Such events are called causally related. Thus the alk invariant often allows one to detect that the events that created the wave fronts are causally related from the current picture of the wave front, without the knowledge of the time-dependent propagation law, times and places of events that created the wave fronts.

First examples relating causality to the linking number were constructed by R Low in the case of $M=\mathbb{R}^{n}$, see [20, [21], 22]. In this case the canonical lifts of the fronts are homologous into the end of $S T \mathbb{R}^{n}$. For such submanifolds the classical linking number can be defined as it was done by S Tabachnikov [30] for $M=\mathbb{R}^{3}$. (This construction of the classical linking number was later generalized by U Kaiser [16] to the case of arbitrary submanifolds of the linking dimension that are homologous into a boundary or into an end of the ambient manifold.) The modified Low conjecture says that two events are causally unrelated if and only if the lifts of their fronts are unlinked in the appropriate sense. For $M$ being a 2-disk with holes strong results proving the conjecture for many cases were obtained by J Natario and P Tod [23].

(b) The classical winding number of a curve around a point $p$ in $\mathbb{R}^{2}$ is the linking number between the curve and the 0 -cycle $\{p, \infty\}$ in $S^{2}=\mathbb{R}^{2} \cup\{\infty\}$. In [8] we considered the generalizations $\operatorname{win}(F, p)$ and $\widehat{\operatorname{win}}(F, p)$ of the winding numbers of the mapping $F: N_{1}^{m-1} \rightarrow M^{m}$ around a point $p:$ pt $=N_{2} \rightarrow M$ to the case where $F_{*}\left(\left[N_{1}\right]\right) \neq 0 \in H_{*}(M)$ and $M$ does not have ends that could play the role of the infinity. (The invariants win and win are the generalizations of the winding number to the case where the observable point $p$ moves and is 
fixed in $M$, respectively.) We showed that affine winding numbers can be effectively used to estimate from below the number of times a wave front has passed through a point between two moments of time.

One can verify that the generalized winding number $\widetilde{\text { win }}$ also is included into the theory explored in this work, if we consider affine linking number for the case $N_{2}=$ pt. (It is clear, see Theorem 7.4 , that in this case $\Omega_{0}(\mathcal{B})=\mathbb{Z}$.)

It is easy to construct the version alk of the invariant alk constructed in this paper, where alk will be a function on $\pi_{0}\left(\mathcal{N}_{1} \times\{*\} \backslash \Sigma\right)$ for some fixed mapping $* \in \mathcal{N}_{2}$. A straightforward verification shows that alk is well-defined provided it takes values in the quotient group of $\Omega_{0}(\mathcal{B})$ by $\operatorname{Im}\left(\mu_{10}: \Omega_{1}\left(\mathcal{N}_{1}\right) \otimes \Omega_{0}\left(\mathcal{N}_{2}\right) \rightarrow\right.$ $\left.\Omega_{0}(\mathcal{B})\right)$. The invariant win constructed in $[8$ is a particular case of such alk where $N_{2}=$ pt.

\subsection{The invariant alk is the universal Vassiliev-Goussarov in- variant of order $\leq 1$}

Fix a natural number $n$. Let $f=\left(f_{1}, f_{2}\right) \in \Sigma \subset \mathcal{N}_{1} \times \mathcal{N}_{2}$ be such that $\operatorname{Im}\left(f_{1}\right) \cap \operatorname{Im}\left(f_{2}\right)$ consists of $n$ distinct double points of transverse intersection. Each double point can be resolved in two essentially different ways: positive and negative, where the sign is as in Definition 3.5. Thus $f$ with $n$ such double points admits $2^{n}$ possible resolutions of the double points. A sign of the resolution is put to be + if the number of negatively resolved double points is even, and it is put to be - otherwise.

Let $\Gamma$ be an abelian group. Let $\alpha$ be a $\Gamma$-valued homotopy link invariant of links from $\mathcal{N}_{1} \times \mathcal{N}_{2} \backslash \Sigma$, ie a function $\alpha: \pi_{0}\left(\mathcal{N}_{1} \times \mathcal{N}_{2} \backslash \Sigma\right) \rightarrow \Gamma$. (Thus $\alpha$ does not change under homotopies of a linked pair that do not involve double points between different components.) The $n$th derivative $\alpha^{(n)}$ of $\alpha$ is a function on singular links with exactly $n$ distinct transverse intersection points between different component (and possibly many self-intersection points of the components). The value of $\alpha^{(n)}$ on such a singular link $f=\left(f_{1}, f_{2}\right)$ is defined as a sum (with appropriate signs) of the values of $\alpha$ on the nonsingular mappings obtained by the $2^{n}$ resolutions of double points. The invariant $\alpha$ is said to be of order $\leq n-1$ (or Vassiliev-Goussarov invariant of order $\leq n-1$, [35, 13, 14]) if $\alpha^{(n)}$ is identically zero.

The invariant alk is an $\mathbb{A}$-valued Vassiliev-Goussarov invariant of order $\leq 1$. To see this consider a singular link $f=\left(f_{1}, f_{2}\right)$ with exactly two transverse double points between different components. We denote by $f( \pm, \pm)$ the four 
nonsingular links obtained by resolutions of the two double points. We denote by $f(\cdot, \pm)$ the singular link with one transverse double point between different components obtained from $f$ by resolving the second singular point and keeping the first singular point intact. To prove that alk is an order $\leq 1$ invariants it suffices to show that

$$
\operatorname{alk}(f(+,+))-\operatorname{alk}(f(+,-))-\operatorname{alk}(f(-,+))+\operatorname{alk}(f(-,-))=0 .
$$

Rewrite this expression as

$$
\begin{aligned}
(\operatorname{alk}(f(+,+))-\operatorname{alk}(f(-,+)))-(\operatorname{alk}(f(+, & -))-\operatorname{alk}(f(-,-))) \\
& =\operatorname{alk}^{(1)}(f(\cdot,+))-\operatorname{alk}^{(1)}(f(\cdot,-)) .
\end{aligned}
$$

By the definition of $\operatorname{alk}^{(1)}$ the values $\operatorname{alk}^{(1)}(f(\cdot,+))$, alk ${ }^{(1)}(f(\cdot,-))$ are equal to $+1[f(\cdot,+)]$, and $+1[f(\cdot,-)] \in \Omega_{0}(\mathcal{B})$. (Here $[f(\cdot,+)],[f(\cdot,-)] \in \pi_{0}(\mathcal{B})$ are the classes of the singular links with one transverse double point.) Clearly $[f(\cdot,+)]=[f(\cdot,-)] \in \pi_{0}(\mathcal{B})$. Thus

$$
\begin{aligned}
\operatorname{alk}(f(+,+))-\operatorname{alk}(f(+,-))-\operatorname{alk}( & f(-,+))+\operatorname{alk}(f(-,-)) \\
& =\operatorname{alk}^{(1)}(f(\cdot,+))-\operatorname{alk}^{(1)}(f(\cdot,-))=0 .
\end{aligned}
$$

Furthermore, if $\alpha: \pi_{0}\left(\mathcal{N}_{1} \times \mathcal{N}_{2} \backslash \Sigma\right) \rightarrow \Gamma$ is a $\Gamma$-valued Vassiliev-Goussarov invariant of order $\leq 1$, then the increment $\alpha^{(1)}\left(\gamma\left(t_{0}\right)\right)=\Delta_{\alpha}\left(\gamma\left(t_{0}\right)\right)$ of $\alpha$ under the positive crossing of $\Sigma$ at $\gamma\left(t_{0}\right) \in \Sigma_{0}$ depends only on the element of $\pi_{0}(\mathcal{B})$ that corresponds to $\gamma\left(t_{0}\right)$. To see this consider a singular link $f$ with two double points obtained from $\gamma\left(t_{0}\right)$ by preserving the existing double point and creating a second double point by a homotopy. Since $\alpha$ is an order $\leq 1$ invariant we have

$$
\begin{aligned}
0 & =\alpha(f(+,+))-\alpha(f(+,-))-\alpha(f(-,+))+\alpha(f(-,-)) \\
& =\alpha^{(1)}(f(\cdot,+))-\alpha^{(1)}(f(\cdot,-)) .
\end{aligned}
$$

Thus the increments into $\alpha$ of the positive crossings of the discriminant at $\alpha^{(1)}(f(\cdot,+))$ and at $\alpha^{(1)}(f(\cdot,-))$ are equal. Clearly we can change $\gamma\left(t_{0}\right) \in \Sigma_{0}$ to any other element in $\Sigma_{0}$ that is in the same component of $\pi_{0}(\mathcal{B})$ by elementary homotopies that pass through an extra double point and by homotopies in $\pi_{0}(\mathcal{B})$ that do not create extra double points between the two components. These operations do not change the value of $\alpha^{(1)}$ on an element in $\mathcal{B}$ and we see that $\alpha^{(1)}\left(\gamma\left(t_{0}\right)\right)$ indeed depends only on the element of $\pi_{0}(\mathcal{B})$ realized by $\gamma\left(t_{0}\right)$.

In particular, there exists a natural homomorphism $B: \Omega_{0}(\mathcal{B}) \rightarrow \Gamma$ that sends the bordism class of $(+1) \gamma\left(t_{0}\right) \in \Omega_{0}(\mathcal{B})$ to $\alpha^{(1)}\left(\gamma\left(t_{0}\right)\right)=\Delta_{\alpha}\left(\gamma\left(t_{0}\right)\right)$. Moreover, 
this homomorphism $B$ passes through the quotient homomorphism $q: \Omega_{0}(\mathcal{B}) \rightarrow$ $\mathbb{A}$ as in 3.7 and therefore we get a homomorphism $A: \mathbb{A} \rightarrow \Gamma$ with $A \circ q=B$, cf Theorem 5.1 below. One verifies that

$$
A\left(\operatorname{alk}(f)-\operatorname{alk}\left(f^{\prime}\right)\right)=\alpha(f)-\alpha\left(f^{\prime}\right),
$$

for all $f, f^{\prime} \in\left(\mathcal{N}_{1}, \times \mathcal{N}_{2} \backslash \Sigma\right)$.

Clearly if $\alpha$ and $\alpha^{\prime}$ are $\Gamma$-valued Vassiliev-Goussarov invariants of order $\leq 1$ such that $\alpha-\alpha^{\prime}$ is a constant mapping, then the corresponding homomorphisms $A$ and $A^{\prime}$ are equal. Let $f_{0} \in \mathcal{N}_{1} \times \mathcal{N}_{2} \backslash \Sigma$ be a chosen preferred point. Then for every $f \in \mathcal{N}_{1} \times \mathcal{N}_{2} \backslash \Sigma$ we have $\alpha(f)=\alpha\left(f_{0}\right)+A(\operatorname{alk}(f))-A\left(\operatorname{alk}\left(f_{0}\right)\right)$. Thus alk completely determines the values of $\alpha$ on all $f$ (modulo $\alpha\left(f_{0}\right)$ ), and hence alk is a universal Vassiliev-Goussarov invariant of order $\leq 1$.

In particular, alk distinguishes all the elements $f, f^{\prime} \in \mathcal{N}_{1} \times \mathcal{N}_{2} \backslash \Sigma$ that can be distinguished via Vassiliev-Goussarov invariants of order $\leq 1$ with values in an arbitrary group $\Gamma$.

\section{Proof of Theorem 3.9}

Definition 4.1 We define $\Sigma_{1}$ to be the subset (stratum) of $\Sigma$ consisting of all the pairs $\left(f_{1}, f_{2}\right)$ such that there exists precisely two pairs of points $y_{1} \in$ $N_{1}, y_{2} \in N_{2}$ as in Definition 3.1. Here we assume that the two double points of the image are distinct. We also choose a base point $*$ of $\mathcal{N}_{1} \times \mathcal{N}_{2}$ with $* \notin \Sigma$.

Notice that $\Sigma_{i}, i=0,1$, is the stratum of codimension $i$ in $\Sigma$. In particular, a generic path in $\mathcal{N}_{1} \times \mathcal{N}_{2}$ intersects $\Sigma_{0}$ in a finite number of points, and a generic disk in $\mathcal{N}_{1} \times \mathcal{N}_{2}$ intersects $\Sigma_{1}$ in a finite number of points.

A generic path $\gamma:[0,1] \rightarrow \mathcal{N}_{1} \times \mathcal{N}_{2}$ that connects two points in $\mathcal{N}_{1} \times \mathcal{N}_{2} \backslash \Sigma$ intersects $\Sigma_{0}$ in finitely many points $\gamma\left(t_{j}\right), j \in J$, and all the intersection points are of the types described in 3.5 Put

$$
\Delta_{\text {alk }}(\gamma)=\sum_{j \in J}\left[\gamma\left(t_{j}\right)\right] \in \Omega_{0}(\mathcal{B})
$$

We let

$$
\begin{aligned}
A & =\left\{(x, y) \in \mathbb{R}^{2} \mid x^{2}+y^{2} \leq 1\right\}, & & B_{1}=\{(x, y) \in A \mid x y=0\}, \\
B_{2} & =\{(x, y) \in A \mid x=0\}, & & B_{3}=\{(0,0)\} \\
B_{4} & =\emptyset & &
\end{aligned}
$$


We define a regular disk in $\mathcal{N}_{1} \times \mathcal{N}_{2}$ to be a generically embedded disk $D$ such that $D \cap \Sigma=D \cap\left(\Sigma_{0} \cup \Sigma_{1}\right)$ and the triple $\left(D, D \cap \Sigma_{0}, D \cap \Sigma_{1}\right)$ is homeomorphic to a triple $(A, B, C), A \supset B \supset C$, where $B$ is one of $B_{i}$ 's and $C$ is equal to $B_{3}$ or $B_{4}$.

Lemma 4.2 Let $\beta$ be a generic loop that bounds a regular disk in $\mathcal{N}_{1} \times \mathcal{N}_{2}$. Then $\Delta_{\text {alk }}(\beta)=0$.

Proof It is easy to see that all the crossings of $\Sigma_{0}$ that happen along $\beta$ can be subdivided into pairs, such that the elements of $\pi_{0}(\mathcal{B})$ corresponding to the two crossings in a pair are equal and the signs of the corresponding crossings of $\Sigma_{0}$ are opposite. Hence the inputs into $\Delta_{\text {alk }}(\beta)$ of the elements of $\Omega_{0}(\mathcal{B})$ corresponding to the two crossings in a pair cancel out and $\Delta_{\text {alk }}(\beta)=0$.

Lemma 4.3 Let $\beta$ be a generic loop that bounds a disk in $\mathcal{N}_{1} \times \mathcal{N}_{2}$. Then $\Delta_{\text {alk }}(\beta)=0$.

Proof Notice that the set $\Sigma \backslash\left(\Sigma_{0} \cup \Sigma_{1}\right)$ is a subset of codimension $\geq 3$ in $\mathcal{N}_{1} \times \mathcal{N}_{2}$. So, without loss of generality we can (using a small deformation of the disk) assume that the disk is the union of regular ones, cf Arnold 1, 2. Now the proof follows from Lemma 4.2.

Corollary 4.4 The invariant $\Delta_{\text {alk }}$ induces a well-defined homomorphism

$$
\Delta_{\text {alk }}: \pi_{1}\left(\mathcal{N}_{1} \times \mathcal{N}_{2}\right)=\pi_{1}\left(\mathcal{N}_{1} \times \mathcal{N}_{2}, *\right) \rightarrow \Omega_{0}(\mathcal{B}) .
$$

Proof Since every element of $\pi_{1}\left(\mathcal{N}_{1} \times \mathcal{N}_{2}, *\right)$ can be represented by a generic loop, the proof follows from Lemma 4.3 .

Now we give another description of $\Delta_{\text {alk }}$. Let $\lambda: \Omega_{1}\left(\mathcal{N}_{1} \times \mathcal{N}_{2}\right) \rightarrow \Omega_{0}(B)$ be the homomorphism from Definition 3.7.

Proposition 4.5 The homomorphism $\Delta_{\text {alk }}: \pi_{1}\left(\mathcal{N}_{1} \times \mathcal{N}_{2}\right) \rightarrow \Omega_{0}(\mathcal{B})$ coincides with the homomorphism

$$
\pi_{1}\left(\mathcal{N}_{1} \times \mathcal{N}_{2}\right) \stackrel{h}{\longrightarrow} \Omega_{1}\left(\mathcal{N}_{1} \times \mathcal{N}_{2}\right) \stackrel{\lambda}{\longrightarrow} \Omega_{0}(\mathcal{B}),
$$

where $h$ is the Hurewicz homomorphism in the bordism theory $\Omega_{*}(-)$ and $\lambda$ is the homomorphism from Definition 3.7. 
Proof Given a generic loop $\alpha$ in $\left(\mathcal{N}_{1}, *\right)$ and the constant loop $e$ in $\left(\mathcal{N}_{2}, *\right)$, let $(\alpha, e)$ be the corresponding loop in $\left(\mathcal{N}_{1} \times \mathcal{N}_{2}, *\right)$. The homotopy class of $(\alpha, e)$ gives us an element $[(\alpha, e)] \in \pi_{1}\left(\mathcal{N}_{1} \times \mathcal{N}_{2}, *\right)$. Similarly, a generic loop $\beta$ in $\left(\mathcal{N}_{2}, *\right)$ gives us an element $[(e, \beta)] \in \pi_{1}\left(\mathcal{N}_{1} \times \mathcal{N}_{2}, *\right)$.

Because of the isomorphism $\pi_{1}\left(\mathcal{N}_{1} \times \mathcal{N}_{2}\right)=\pi_{1}\left(\mathcal{N}_{1}\right) \times \pi_{1}\left(\mathcal{N}_{2}\right)$, the classes $[(\alpha, e)]$ and $[(e, \beta)]$ generate the group $\pi_{1}\left(\mathcal{N}_{1} \times \mathcal{N}_{2}, *\right)$. So, since $\Delta_{\text {alk }}$ is a homomorphism, it suffices to prove that $\Delta_{\text {alk }}[(\alpha, e)]=(\lambda \circ h)[(\alpha, e)]$, and similarly for $[(e, \beta)]$. We do it here for the loops $(\alpha, e)$ only.

We calculate $\Delta_{\text {alk }}[(\alpha, e)] \in \Omega_{0}(\mathcal{B})$. Fix a mapping $\bar{e}: N_{2} \rightarrow M$ in $\mathcal{N}_{2}$ and consider the mapping

$$
\bar{\alpha}: S^{1} \times\left(N_{1} \sqcup N_{2}\right) \rightarrow M
$$

such that $\left.\bar{\alpha}\right|_{S^{1} \times N_{1}}=\alpha$ and $\left.\bar{\alpha}\right|_{S^{1} \times N_{2}}$ coincides with the composition

$$
S^{1} \times N_{2} \stackrel{\text { projection }}{\longrightarrow} N_{2} \stackrel{\bar{e}}{\longrightarrow} M .
$$

Without loss of generality we may assume that $\left.\bar{\alpha}\right|_{S^{1} \times N_{1}}$ is transverse to $\bar{e}$. The inclusion $\mathcal{N}_{1} \rightarrow \mathcal{N}_{1} \times \mathcal{N}_{2}, x \mapsto(x, e)$ allows us to regard $h(\alpha) \in \Omega_{1}\left(\mathcal{N}_{1}\right)$ as an element of $\Omega_{1}\left(\mathcal{N}_{1} \times \mathcal{N}_{2}\right)$.

Now it is easy to see that

$$
\Delta_{\text {alk }}[(\alpha, e)]=\mu_{10}\left(\left[\left.\bar{\alpha}\right|_{S^{1} \times N_{1}}\right] \otimes[\bar{e}]\right)=\lambda(h(\alpha)) \in \Omega_{1}(\mathcal{B}),
$$

where $\left[\left.\bar{\alpha}\right|_{S^{1} \times N_{1}}\right]$ and $[\bar{e}]$ are the bordism classes of corresponding maps.

\section{Corollary 4.6}

$$
\operatorname{Im}\left\{\Delta_{\text {alk }}: \pi_{1}\left(\mathcal{N}_{1} \times \mathcal{N}_{2}, *\right) \rightarrow \Omega_{0}(\mathcal{B})\right\}=\operatorname{Indet} \subset \Omega_{0}(\mathcal{B}) .
$$

Take an arbitrary point $f=\left(f_{1}^{1}, f_{2}^{1}\right) \in \mathcal{N}_{1} \times \mathcal{N}_{2} \backslash \Sigma$ and choose a generic path $\gamma$ going from $*$ to $f$. We set

$$
\operatorname{alk}(f)=q\left(\Delta_{\text {alk }}(\gamma)\right) \in \mathbb{A}
$$

where $q$ is the epimorphism from Definition 3.7

Theorem 4.7 The function

$$
\text { alk: } \mathcal{N}_{1} \times \mathcal{N}_{2} \backslash \Sigma \rightarrow \mathbb{A}
$$

is an affine linking invariant. Furthermore, any other affine linking invariant alk coincides with alk if $\widetilde{\operatorname{alk}}(*)=0$. 
Proof To show that alk is constant on path components we must verify that the definition of alk is independent on the choice of the path $\gamma$ that goes from * to $f$. This is the same as to show that $q\left(\Delta_{\text {alk }}(\varphi)\right)=0$ for every closed generic loop $\varphi$ at $*$. But this follows from Corollary 4.6] directly.

Furthermore, it is clear that alk increases by $q([\gamma(t)]) \in \mathbb{A}$ under a transverse passage by a path $\gamma$ through the stratum $\Sigma_{0}$ at the point $\gamma(t)$. This yields property (b) of alk from Theorem 3.9. The last claim is obvious.

Remark 4.8 Clearly alk depends on the choice of $*$. On the other hand, if we change $*$, then new alk and the old alk will differ by an additive constant. This is the reason why we use the adjective "affine".

Clearly, Theorem 3.9 is a direct consequence of Theorem 4.7

\section{Relations between alk and the classical linking in- variant $1 \mathrm{k}$}

Given a closed oriented manifold $N^{n}$ with the fundamental class $[N] \in H_{n}(M)$, we say that a map $f: N \rightarrow M$ is zero-homologous if $f_{*}([N])=0 \in H_{n}(M)$.

Let $\varepsilon: \Omega_{0}(\mathcal{B}) \rightarrow \mathbb{Z}$ be the homomorphism from (3.1).

Theorem 5.1 Suppose that $\mathcal{N}_{1}$ and $\mathcal{N}_{2}$ consist of zero-homologous mappings. Then $\varepsilon$ (Indet) $=0$. Furthermore, for all $f=\left(f_{1}, f_{2}\right), f^{\prime}=\left(f_{1}^{\prime}, f_{2}^{\prime}\right) \in \mathcal{N}=$ $\mathcal{N}_{1} \times \mathcal{N}_{2} \backslash \Sigma$, we have

$$
\varepsilon(\operatorname{alk}(f))-\varepsilon\left(\operatorname{alk}\left(f^{\prime}\right)\right)=\operatorname{lk}\left(f_{1}, f_{2}\right)-\operatorname{lk}\left(f_{1}^{\prime}, f_{2}^{\prime}\right) \in \mathbb{Z} .
$$

Proof Since $\mathcal{N}_{1}$ and $\mathcal{N}_{2}$ consist of zero-homologous maps, the classical linking invariant lk: $\mathcal{N}_{1} \times \mathcal{N}_{2} \backslash \Sigma \rightarrow \mathbb{Z}$ is well-defined. Now, similarly to $\Delta_{\text {alk }}$, we define

$$
\Delta_{\mathrm{lk}}: \pi_{1}\left(\mathcal{N}_{1} \times \mathcal{N}_{2}, *\right) \rightarrow \mathbb{Z}, \quad \Delta_{\mathrm{lk}}(\gamma)=\sum_{i=1}^{k} \sigma\left(\gamma, t_{i}\right),
$$

where the generic loop $\gamma$ in $\left(\mathcal{N}_{1} \times \mathcal{N}_{2}, *\right)$ intersects $\Sigma_{0} \subset \Sigma \subset \mathcal{N}_{1} \times \mathcal{N}_{2}$ in certain points $\gamma\left(t_{1}\right), \ldots, \gamma\left(t_{k}\right)$. (Here we use the notation $\gamma$ for the loop as well as for its homotopy class.) Since lk is well-defined, we conclude that $\Delta_{\mathrm{lk}}(\gamma)=0$ for all $\gamma$, ie $\sum \sigma\left(\gamma, t_{i}\right)=0$. 
Now, we have

$$
\Delta_{\text {alk }}([\gamma])=\sum\left[\gamma\left(t_{i}\right)\right] \in \Omega_{0}(\mathcal{B})
$$

So, in view of (3.1)

$$
\varepsilon\left(\Delta_{\text {alk }}([\gamma])\right)=\sum \varepsilon\left(\left[\gamma\left(t_{i}\right)\right]\right)=\sum \sigma\left(\gamma, t_{i}\right)=0 .
$$

Thus, by Corollary 4.6,

$$
\varepsilon(\text { Indet })=\varepsilon\left(\operatorname{Im}\left(\Delta_{\text {alk }}\right)\right)=0 .
$$

Now take a generic path $\gamma$ which connects $f$ and $f^{\prime}$. Then

$$
\begin{aligned}
\varepsilon(\operatorname{alk}(f))-\varepsilon\left(\operatorname{alk}\left(f^{\prime}\right)\right) & =\varepsilon\left(\sum\left[\gamma\left(t_{i}\right)\right]\right)=\sum \sigma\left(\gamma, t_{i}\right) \\
& =\operatorname{lk}\left(f_{1}, f_{2}\right)-\operatorname{lk}\left(f_{1}^{\prime}, f_{2}^{\prime}\right),
\end{aligned}
$$

which proves the second claim of the theorem.

Remarks 5.2 Theorem 5.1 demonstrates that, up to an additive constant, $\varepsilon \circ$ alk is equal to the classical linking number lk whenever $\mathcal{N}_{1}$ and $\mathcal{N}_{2}$ consist of zero-homologous mappings. So, alk is an extension of the classical lk-invariant of zero-homologous submanifolds.

Since the homomorphism $\varepsilon$ is the summation over the components of $\mathcal{B}$, we conclude that, for zero-homologous mappings, alk can be regarded as a splitting of the classical linking invariant into a collection of independent invariants. In many cases it can be shown that $\Omega_{0}(\mathcal{B})$ is an infinitely generated abelian group (see Theorem [7.4-Corollary 17.10) and that the indeterminacy subgroup Indet is zero (see Section 6). Since it is easy to show (see Corollary [7.5) that the mapping alk: $\pi_{0}\left(\mathcal{N}_{1} \times \mathcal{N}_{2} \backslash \Sigma\right) \rightarrow \mathbb{A}$ is always surjective, we see that for these cases the classical lk invariant of zero-homologous submanifolds splits into infinitely many independent invariants.

On the other hand, as it was explained in this paper, the invariant alk exists regardless of whether the mappings are zero-homologous or not.

Also, there are many cases where $\mathcal{N}_{1}, \mathcal{N}_{2}$ do not consist of zero-homologous mappings while nevertheless $\varepsilon$ (Indet) $=0=$ Indet, and thus $\varepsilon \circ$ alk is a $\mathbb{Z}^{-}$ valued invariant.

\section{Examples where the indeterminacy subgroup van- ishes}

Given the manifolds $M, N_{1}, N_{2}$ as in Section 3 , we assume in addition that $N_{1}$ and $N_{2}$ are connected, and that $n_{1} n_{2}>0$. 
Theorem 6.1 (Preissman) Let $M$ be a closed manifold that admits a Riemannian metric of negative sectional curvature. Then the following holds:

(i) Every nontrivial abelian subgroup of $\pi_{1}(M)$ is an infinite cyclic group.

(ii) For every nontrivial abelian subgroup $A$ of $\pi$ there exists a unique abelian subgroup $B_{A}$ of $\pi$ which contains $A$ and is maximal with respect to this property. In fact, $B_{A}$ is the centralizer $Z(A)$ of $A$ in $\pi_{1}(M)$.

Proof See do Carmo [1] or the original paper by Preissman [24].

Definition 6.2 A finitely generated group $\pi$ is called a Preissman group if it satisfies the properties (i) and (ii) from Theorem 6.1

Proposition 6.3 Let $\pi$ be a Preissman group. Let $\alpha, \beta \in \pi$ be such that $\alpha \beta \neq \beta \alpha$, and let $\gamma \in \pi$ be such that $\alpha \gamma=\gamma \alpha$ and $\beta \gamma=\gamma \beta$. Then $\gamma=e$.

Proof Suppose that $\gamma \neq e$. Let $G=\{x\}$ be the (unique) maximal cyclic subgroup of $\pi$ which contains $\gamma$. Since $\alpha \gamma=\gamma \alpha$, the subgroup $\{\alpha, \gamma\}$ of $\pi$ is contained in $G$, and so $\alpha=x^{m}$ for some $m$. Similarly, $\beta=x^{k}$, and thus $\alpha \beta=\beta \alpha$. This is a contradiction.

Theorem 6.4 For $M, N_{1}, N_{2}$ as above, suppose that $\pi_{1}(M)$ is a Preissman group, and that $\pi_{i}(M)=0$ for $2 \leq i \leq 1+\max \left\{n_{1}, n_{2}\right\}$. Then the indeterminacy subgroup Indet $\subset \Omega_{0}(\mathcal{B})$ is the zero subgroup, Indet $=\{0\} \subset \Omega_{0}(\mathcal{B})$.

Proof Throughout the proof we denote $\pi_{1}(M)$ by $\pi$. We must prove that, for every $\alpha \in \pi_{1}\left(\mathcal{N}_{1}\right)$ and $\beta \in \pi_{1}\left(\mathcal{N}_{2}\right)$,

$$
\Delta_{\text {alk }}[(\alpha, e)]=0=\Delta_{\text {alk }}[(e, \beta)]
$$

(see Lemma 4.6).

We prove the first equality from (6.1) only, the second equality can be proved in the similar way. Fix $\phi_{i} \in \mathcal{N}_{i}$ for $i=1,2$, and consider a loop $\alpha$ in $\left(\mathcal{N}_{1}, \phi_{1}\right)$. Let $\widetilde{\alpha}: S^{1} \times N_{1} \rightarrow M$ be the adjoint map, $\widetilde{\alpha}(t, n)=\alpha\left(t(n)\right.$. Since $\pi_{i}(M)=0$ for $2 \leq i \leq 1+n_{1}$, it follows from the elementary obstruction theory that the homomorphism

$$
\widetilde{\alpha}_{*}: \pi_{1}\left(S^{1} \times N\right) \rightarrow \pi
$$

completely determines the homotopy class of $\widetilde{\alpha}$. We use the isomorphism $\pi_{1}\left(S^{1} \times N\right) \simeq \pi_{1}\left(S^{1}\right) \times \pi_{1}(N)$ and set

$$
\gamma=\widetilde{\alpha}_{*}(\iota, e)
$$

where $\iota \in \pi_{1}\left(S^{1}\right)$ is the generator. 
Lemma 6.5 If $\gamma=e$, then $\Delta_{\text {alk }}[(\alpha, e)]=0$.

Proof Indeed, in this case $\widetilde{\alpha}: S^{1} \times N_{1} \rightarrow M$ is homotopic to the map

$$
S^{1} \times N_{1} \stackrel{\text { proj }}{\longrightarrow} N_{1} \stackrel{\phi_{1}}{\longrightarrow} M,
$$

because both maps induce the same homomorphism of fundamental groups. So, since $n_{1}+n_{2}=m-1<m$, there is a generic map $\widehat{\alpha}$ homotopic to $\widetilde{\alpha}$ such that $\widehat{\alpha}\left(S^{1} \times N_{1}\right)$ does not meet $\phi_{2}\left(N_{2}\right)$.

Lemma 6.6 If $\operatorname{Im} \widetilde{\alpha}_{*}=\mathbb{Z} \subset \pi$, then $\Delta_{\text {alk }}[(\alpha, e)]=0$.

Proof If $\operatorname{Im} \widetilde{\alpha}_{*}=\mathbb{Z}$, then $\widetilde{\alpha}_{*}$ can be decomposed as

$$
\pi_{1}\left(S^{1} \times N_{1}\right) \rightarrow \mathbb{Z} \subset \pi .
$$

Since $S^{1}=K(\mathbb{Z}, 1)$, the map $\pi_{1}\left(S^{1} \times N_{1}\right) \rightarrow \mathbb{Z}$ in (6.3) can be induced by a map $\varphi: S^{1} \times N_{1} \rightarrow S^{1}$. Furthermore, the inclusion $\mathbb{Z} \subset \pi$ in (6.3) can be induced by a map (inclusion) $\psi: S^{1} \rightarrow M$, and we can assume that $\psi\left(S^{1}\right)$ does not meet $\phi_{2}\left(N_{2}\right)$ since $m-n_{2}>1$. Now, the map $\widetilde{\alpha}: S^{1} \times N_{1} \rightarrow M$ is homotopic to a map

$$
\widehat{\alpha}: S^{1} \times N_{1} \stackrel{\varphi}{\longrightarrow} S^{1} \stackrel{\psi}{\longrightarrow} M .
$$

Clearly, $\widehat{\alpha}$ does not meet $\phi_{2}\left(N_{2}\right)$ and, moreover, any small perturbation of $\widehat{\alpha}$ does not. Thus, $\Delta_{\text {alk }}[(\alpha, e)]=0$.

Now we finish the proof of Theorem 6.4 Consider the homomorphism

$$
\left(\phi_{1}\right)_{*}: \pi_{1}\left(N_{1}\right) \rightarrow \pi \text {. }
$$

If $\operatorname{Im}\left(\phi_{1}\right)_{*}$ is non-abelian, then $\gamma=e \in \pi$ by Proposition 6.3 because $\gamma$ commutes with $\operatorname{Im}\left(\phi_{1}\right)_{*}$. So, $\Delta_{\text {alk }}[(\alpha, e)]=0$ by Lemma 6.5.

Furthermore, assume that $\operatorname{Im}\left(\phi_{1}\right)_{*}$ is abelian. Since $\gamma$ commutes with $\operatorname{Im}\left(\phi_{1}\right)_{*}$ we conclude that $\operatorname{Im}\left(\widetilde{\alpha}_{*}\right)$ is an abelian subgroup of $\pi$. So, $\operatorname{Im}\left(\widetilde{\alpha}_{*}\right)=\mathbb{Z}$ or 0 because $\pi$ is a Preissman group. If $\operatorname{Im}\left(\widetilde{\alpha}_{*}\right)=\mathbb{Z}$ then $\Delta_{\text {alk }}[(\alpha, e)]=0$ (by Lemma [6.6), and if $\operatorname{Im}\left(\widetilde{\alpha}_{*}\right)=0$, then $\gamma=e \in \pi$ and $\Delta_{\text {alk }}[(\alpha, e)]=0$ (by Lemma 6.5).

Remark 6.7 Assume that $n_{1}+n_{2}+1 \neq m$ but otherwise the manifolds $N_{1}, N_{2}, M$ satisfy all the conditions of Theorem 6.4. Assume moreover that $\max \left(n_{1}, n_{2}\right)<m-1$ and that there exists $\left(f_{1}, f_{2}\right) \in \mathcal{N}_{1} \times \mathcal{N}_{2}$ with $\operatorname{Im} f_{1} \cap$ $\operatorname{Im} f_{2}=\emptyset$. Then the straightforward modifications of the proof of the Theorems 6.4 shows that $\operatorname{Im} \mu_{1,0}=\operatorname{Im} \mu_{0,1}=0 \subset \Omega_{n_{1}+n_{2}+1+0-m}(\mathcal{B})$. 
Theorem 6.8 Let $M, N_{1}$ and $N_{2}$ be as above with an extra condition that $\pi_{i}(M)$ is finite for $i=1, \ldots, \max \left(n_{1}, n_{2}\right)+1$, then the indeterminacy subgroup Indet $\subset \Omega_{0}(\mathcal{B})$ is the zero subgroup.

Proof Elementary obstruction theory implies that a mapping $N_{i} \times * \rightarrow M$ (for $i=1,2$ ) has are finitely many non-homotopic extensions to a mapping $N_{i} \times S^{1} \rightarrow M$ (for $\left.i=1,2\right)$. Thus $\pi_{1}\left(\mathcal{N}_{1} \times \mathcal{N}_{2}\right)$ is finite and hence

$$
\operatorname{Im}\left(\Delta_{\text {alk }}: \pi_{1}\left(\mathcal{N}_{1} \times \mathcal{N}_{2}, *\right) \rightarrow \Omega_{0}(\mathcal{B})\right)=\operatorname{Indet} \subset \Omega_{0}(\mathcal{B})
$$

is a finite subgroup. Since $\Omega_{0}(\mathcal{B})$ is torsion free, we get that Indet $=0$.

Remark 6.9 Assume that $n_{1}+n_{2}+1 \neq m$ but otherwise the manifolds $N_{1}, N_{2}, M$ satisfy all the conditions of Theorem 6.8. Then the straightforward modification of the proof of Theorem 6.8 shows that $\operatorname{Im} \mu_{1,0}=\operatorname{Im} \mu_{0,1}=0 \subset$ $\Omega_{n_{1}+n_{2}+1+0-m}(\mathcal{B})$.

Examples 6.10 (1) It is well-known that every closed manifold $M^{m}$ that admits a complete Riemannian metric of negative sectional curvature has the universal covering space homeomorphic to $\mathbb{R}^{m}$, and thus $\pi_{i}(M)=0$, for $i>1$, while $\pi_{1}(M)$ is a Preissman group.

Combining this with Theorems 6.1 and 6.4 we get that for such $M$ the group Indet $=0$ for all $N_{1}, N_{2}, \mathcal{N}_{1}, \mathcal{N}_{2}$, and hence $\mathbb{A}$ is a free abelian group.

To construct more manifolds with Preissman fundamental group, notice that the total space of a locally trivial bundle $F \rightarrow E \rightarrow B$ has the Preismann fundamental group if $F$ is simply-connected and $\pi_{1}(B)$ is Preissman.

(2) Let $M^{m}$ be an oriented base space of a finite covering map $\Sigma^{m} \rightarrow M$, where $\Sigma^{m}$ is a simply connected $m$-dimensional rational homology sphere. The Serre-Hurewicz theorem implies that $\pi_{k}\left(\Sigma^{m}\right)$ is finite, for $k=2, \ldots, m-1$, and therefore $\pi_{k}(M)$ is finite, for $k=1, \ldots, m-1$. Now Theorem 6.8 implies that Indet $=0$ for all $N_{1}, N_{2}, \mathcal{N}_{1}, \mathcal{N}_{2}$, provided that $n_{1}, n_{2}>0$.

Theorem 5.1 implies that in all these cases, if $\mathcal{N}_{1}, \mathcal{N}_{2}$ consist of zero-homologous mappings, then alk is a splitting of the classical linking invariant $\mathrm{k}$ into a direct sum of independent $\mathbb{Z}$-valued invariants. Corollary 7.10 says that this direct sum is infinite when $M$ is closed and admits a complete metric of negative sectional curvature; the images of $\pi_{1}\left(N_{1}\right), \pi_{1}\left(N_{2}\right)$ in $\pi_{1}(M)$ under the homomorphisms induced by the mappings $f_{1} \in \mathcal{N}_{1}, f_{2} \in \mathcal{N}_{2}$ are nontrivial; and $\left|H_{1}(M) /\left(\operatorname{Im} f_{1 *}\left(H_{1}\left(N_{1}\right)\right)+\operatorname{Im} f_{2 *}\left(H_{1}\left(N_{2}\right)\right)\right)\right|=\infty$. 


\section{$7 \quad$ Description of $\pi_{0}(\mathcal{B})$ and $\Omega_{0}(\mathcal{B})$}

In Section 17 we assume $N_{1}$ and $N_{2}$ are connected; however we do not assume that $n_{1}+n_{2}+1=m$. The goal of this section is to give a method of evaluation of $\pi_{0}(\mathcal{B})$ (and therefore of $\Omega_{0}(\mathcal{B})$ since the last group is the free abelian group with the base $\left.\pi_{0}(\mathcal{B})\right)$ and, in particular, to demonstrate that $\pi_{0}(\mathcal{B})$ can be infinite in spite of $\pi_{0}\left(\mathcal{N}_{1}\right)$ and $\pi_{0}\left(\mathcal{N}_{2}\right)$ being one-point sets.

We must recall some facts from elementary homotopy theory. Given two pointed spaces $X$ and $Y$, let $[X, Y]^{\bullet}$ and $[X, Y]$ be the set of pointed homotopy classes of pointed maps $X \rightarrow Y$ and the set of unpointed homotopy classes, respectively. Then $\pi_{1}(Y)$ acts on $[X, Y]^{\bullet}$ in a usual way, and

$$
[X, Y]^{\bullet} / \pi_{1}(Y)=[X, Y]
$$

(see [27], for example).

Furthermore, $\pi_{1}(X)$ acts on $[X, X]$, and therefore we get a right $\pi_{1}(X)$-action on $[X, Y]$ via the composition map $[X, X] \times[X, Y] \rightarrow[X, Y]$. In greater detail, we apply $\alpha \in \pi_{1}(X)$ to the homotopy class $1_{X} \in[X, X]$ and get a map (homotopy class) $\alpha\left(1_{X}\right)$. Now, given $f \in[X, Y]$, we define $f \alpha=(f) \alpha \in[X, Y]$ to be the composition $f \circ \alpha\left(1_{X}\right)$. It is worthy to mention that, for the standard $\pi_{1}(Y)$-action on $[X, Y]$ we have

$$
\beta(f)=\beta\left(1_{Y}\right) \circ f, \quad \beta \in \pi_{1}(Y), f \in[X, Y] .
$$

Furthermore, given $\alpha \in \pi_{1}(X)$ and $f \in[X, Y]^{\bullet}$, we have

$$
f \alpha=f_{*}(\alpha) f
$$

and so any orbit of the (right) $\pi_{1}(X)$-action is contained in an orbit of the $\pi_{1}(Y)$-action.

Lemma 7.1 The $\pi_{1}(Y)$-action on $[X, Y]^{\bullet}$ and the $\pi_{1}(X)$-action on $[X, Y]^{\bullet}$ commute.

Proof Choose $\alpha \in \pi_{1}(X), \beta \in \pi_{1}(Y)$ and consider the composition

$$
X \stackrel{\alpha\left(1_{X}\right)}{\longrightarrow} X \stackrel{f}{\longrightarrow} Y \stackrel{\beta\left(1_{Y}\right)}{\longrightarrow} Y .
$$

Then, in view of (7.2), we have

$$
(\beta(f)) \alpha=\left(\beta\left(1_{Y}\right) \circ f\right) \circ \alpha\left(1_{X}\right)=\beta\left(1_{Y}\right) \circ\left(f \circ \alpha\left(1_{X}\right)\right)=\beta((f) \alpha) .
$$

Now, consider two pointed spaces $X_{1}$ and $X_{2}$ and the actions

$$
\left[X_{1}, Y\right]^{\bullet} \times \pi_{1}\left(X_{1}\right) \rightarrow\left[X_{1}, Y\right]^{\bullet} \quad \text { and } \quad\left[X_{2}, Y\right]^{\bullet} \times \pi_{1}\left(X_{2}\right) \rightarrow\left[X_{2}, Y\right]^{\bullet} .
$$


Together these actions yield the action

$$
\left(\left[X_{1}, Y\right]^{\bullet} \times\left[X_{2}, Y\right]^{\bullet}\right) \times\left(\pi_{1}\left(X_{1}\right) \times \pi_{1}\left(X_{2}\right)\right) \rightarrow\left[X_{1}, Y\right]^{\bullet} \times\left[X_{2}, Y\right]^{\bullet} .
$$

Because of Lemma 7.1] this action commutes with the diagonal $\pi_{1}(Y)$-action on $\left[X_{1}, Y\right]^{\bullet} \times\left[X_{2}, Y\right]^{\bullet}$.

Remark 7.2 Since $\left[X_{1}, Y\right]^{\bullet} \times\left[X_{2}, Y\right]^{\bullet}=\left[X_{1} \vee X_{2}, Y\right]^{\bullet}$, we have the action of the group $\pi_{1}\left(X_{1} \vee X_{2}\right)=\pi_{1}\left(X_{1}\right) * \pi_{1}\left(X_{2}\right)$ on $\left[X_{1}, Y\right]^{\bullet} \times\left[X_{2}, Y\right]^{\bullet}$. Let $i: \pi_{1}\left(X_{1}\right) \subset \pi_{1}\left(X_{1}\right) * \pi_{1}\left(X_{2}\right)$ be the standard inclusion. We emphasize that the $i\left(\pi_{1}\left(X_{1}\right)\right)$-action on $\left[X_{1}, Y\right]^{\bullet} \times\left[X_{2}, Y\right]^{\bullet}$ does not coincide with the action coming from (7.4).

Given $f \in[X, Y]^{\bullet}$, let

$$
S_{f}=\left\{\alpha \in \pi_{1}(Y) \mid \alpha f=f\right\}
$$

Proposition 7.3 $S_{f} \subset Z\left(f_{*}\left(\pi_{1}(X)\right)\right.$, where $Z G$ denotes the centralizer of $G$ in $\pi_{1}(Y)$.

Proof Let $H$ be the set of homomorphisms $\pi_{1}(X) \rightarrow \pi_{1}(Y)$ induced by pointed maps $X \rightarrow Y$. The $\pi_{1}(Y)$-action on $[X, Y]^{\bullet}$ induces the $\pi_{1}(Y)$-action on $H$. Here for $\alpha \in \pi_{1}(Y)$ we have

$$
\left(\alpha\left(f_{*}\right)\right)(u)=\alpha\left(f_{*}(u)\right) \alpha^{-1}
$$

where $u \in \pi_{1}(X)$ and $f: X \rightarrow Y$. Thus, if $\alpha \in S_{f}$ then $\left(\alpha\left(f_{*}\right)\right)(u)=$ $\alpha\left(f_{*}(u)\right) \alpha^{-1}=f_{*}(u)$, ie $\alpha \in Z\left(f_{*}\left(\pi_{1}(X)\right)\right.$.

Choose base points in $N_{1}, N_{2}$ and $M$ and let $\mathcal{N}_{i}^{\bullet}, i=1,2$, be the space of all pointed maps $f: N_{i} \rightarrow M$ such that the unpointed map $f$ belongs to $\mathcal{N}_{i}$. Clearly, the subset $\pi_{0}\left(\mathcal{N}_{i}^{\bullet}\right)$ of $\left[N_{i}, M\right]^{\bullet}$ is invariant with respect to $\pi_{1}\left(N_{i}\right)$ - and $\pi_{1}(M)$-actions. Moreover, because of (17.1),$\pi_{0}\left(\mathcal{N}_{i}^{\bullet}\right)$ is the orbit of $\pi_{1}(M)-$ action on $\left[N_{i}, M\right]^{\bullet}$.

Clearly, the bijection $\left[N_{1} \vee N_{2}, M\right]^{\bullet}=\left[N_{1}, M\right]^{\bullet} \times\left[N_{2}, M\right]^{\bullet}$ converts the $\pi_{1}(M)-$ action on $\left[N_{1} \vee N_{2}, M\right]$ into the diagonal action on $\left[N_{1}, M\right]^{\bullet} \times\left[N_{2}, M\right]^{\bullet}$.

\section{Theorem 7.4}

$$
\pi_{0}(\mathcal{B})=\left(\pi_{1}\left(N_{1}\right) \times \pi_{1}\left(N_{2}\right)\right) \backslash\left(\pi_{0}\left(\mathcal{N}_{1}^{\bullet}\right) \times \pi_{0}\left(\mathcal{N}_{2}^{\bullet}\right)\right) / \pi_{1}(M) .
$$


Proof Consider the inclusion $j: \mathcal{N}_{1}^{\bullet} \times \mathcal{N}_{2} \bullet \rightarrow \mathcal{B}$. Since $M$ and $N_{i}$ are connected, the map $j_{*}: \pi_{0}\left(\mathcal{N}_{1} \bullet \times \mathcal{N}_{2}^{\bullet}\right) \rightarrow \pi_{0}(\mathcal{B})$ is surjective. Now, consider two points $b, b^{\prime} \in \mathcal{N}_{1}^{\bullet} \times \mathcal{N}_{2}^{\bullet}$ and set $j(b)=\left(\rho_{1}, \rho_{2}, \phi_{1}, \phi_{2}\right)$ and $j\left(b^{\prime}\right)=$ $\left(\rho_{1}^{\prime}, \rho_{2}^{\prime}, \phi_{1}^{\prime}, \phi_{2}^{\prime}\right) \in \mathcal{B}$. Then $\phi_{1} \rho_{1}=\phi_{1}^{\prime} \rho_{1}^{\prime}$. Suppose that there is a path from $b$ to $b^{\prime}$ in $\mathcal{B}$. Consider this path as a quadruple $\left(\rho_{1}(t), \rho_{2}(t), \phi_{1}(t), \phi_{2}(t)\right), t \in I$. Now, the loop $\rho_{i}(t)$ gives us the loop (homotopy class) $\alpha \in \pi_{1}\left(N_{i}\right), i=1,2$, while the loop

$$
\left(\phi_{1}(t)\right)\left(\rho_{1}(t)\right)=\left(\phi_{2}(t)\right)\left(\rho_{2}(t)\right)
$$

gives us an element $\beta \in \pi_{1}(M)$. Clearly, $\beta([b])\left(\alpha_{1}, \alpha_{2}\right)=\left[b^{\prime}\right]$ where $[b]$ and $\left[b^{\prime}\right]$ are components of $\mathcal{N}_{1}^{\bullet} \times \mathcal{N}_{2}^{\bullet}$ containing $b$ and $b^{\prime}$, respectively.

Corollary 7.5 The mapping alk: $\pi_{0}\left(\mathcal{N}_{1} \times \mathcal{N}_{2} \backslash \Sigma\right) \rightarrow \mathbb{A}$ of Theorem 3.9 is surjective.

Proof Take an arbitrary point $b \in \mathcal{B}$ and consider the class $[b] \in \Omega_{0}(\mathcal{B})$. Clearly it suffices to show that, given $\left(f_{1}, f_{2}\right) \in \mathcal{N}_{1} \times \mathcal{N}_{2} \backslash \Sigma$, there exist generic paths $\gamma_{i}: I \rightarrow \mathcal{N}_{1} \times \mathcal{N}_{2}, i=1,2$, starting at $\left(f_{1}, f_{2}\right)$ such that $\gamma_{i}(t)$ intersects $\Sigma_{0}$ for exactly one value $t_{i} \in(0,1), i=1,2$, and $\left[\gamma_{1}\left(t_{1}\right)\right]=[b],\left[\gamma_{2}\left(t_{2}\right)\right]=-[b]$. Clearly there exists $b^{\prime}=\left[\phi_{1}, \phi_{2}, \rho_{1}, \rho_{2}\right] \in \mathcal{B}$ such that $\pm[b]^{\prime}=[b]$ and that one of the two possible resolutions of the double point between $\phi_{1}$ and $\phi_{2}$ is isotopic to $\left(f_{1}, f_{2}\right)$. Let $\left(\phi_{1}^{\prime}, \phi_{2}^{\prime}\right)$ be this resolution and let $\tilde{\gamma}$ be the isotopy path that connects $\left(f_{1}, f_{2}\right)$ to $\left(\phi_{1}^{\prime}, \phi_{2}^{\prime}\right)$.

It is easy to construct (see Figure 1) a local deformation $\tilde{\gamma}_{+}$(respectively, $\tilde{\gamma}_{-}$) of $\left(\phi_{1}^{\prime}, \phi_{2}^{\prime}\right)$ that involves only one passage through a double point and such that the sign of the corresponding crossing of $\Sigma_{0}$ is positive (respectively, negative) and the associated element of $\pi_{0}(\mathcal{B})$ is the same as the one for $b^{\prime}$. The desired paths $\gamma_{i}$ are the paths $\tilde{\gamma}_{+} \tilde{\gamma}$ and $\tilde{\gamma}_{-} \tilde{\gamma}$.

Corollary 7.6 Assume that $N_{1}$ and $N_{2}$ are simply connected and that $\pi_{1}(M)$ is infinite. Also, assume that, for both $i=1$ and $i=2$, the $\pi_{1}(M)$-action on $\pi_{0}\left(\mathcal{N}_{i}^{\bullet}\right)$ is free. Then $\pi_{0}(B)$ is an infinite set.

Example 7.7 Let $N_{1}=N_{2}=S^{n}$, and let $M$ be a manifold which has the $(n+2)$-type of the wedge $S^{1} \vee S^{n}$. Fix a map $f: S^{n} \rightarrow M$ which induces an isomorphism in $n$-dimensional homology. Let $\mathcal{N}=\mathcal{N}_{1}=\mathcal{N}_{2}$ be the path component of $f$ in the space of smooth maps $S^{n} \rightarrow M$. Then $\pi_{0}(B)$ is an infinite set because of Corollary [7.6. 

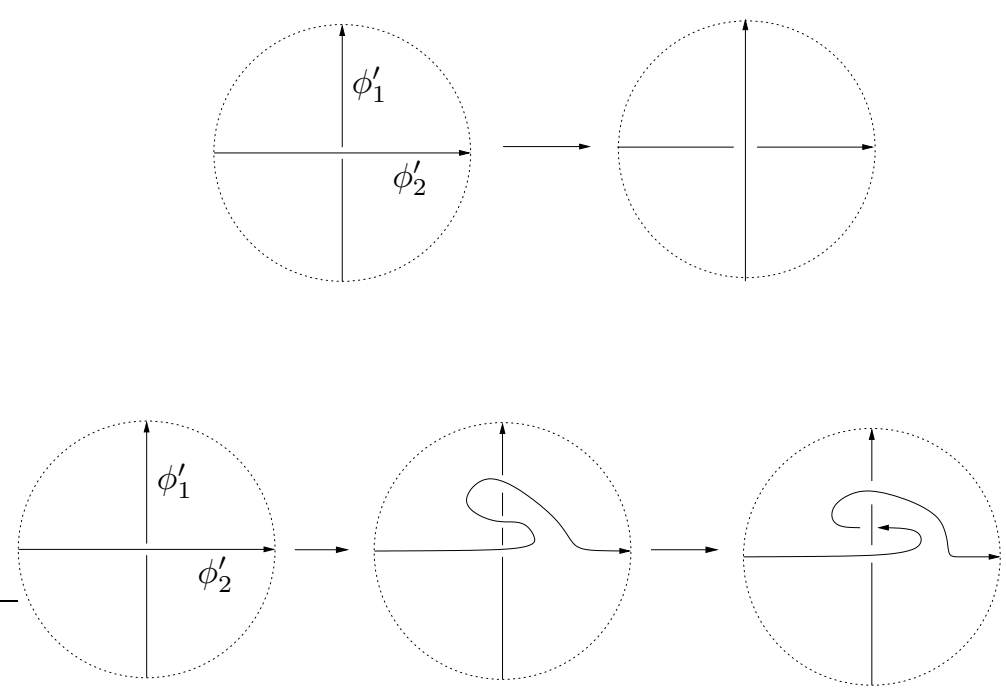

Figure 1: The paths $\tilde{\gamma}_{+}$and $\tilde{\gamma}_{-}$in the 1 -dimensional case

Another series of cases with $\pi_{0}(\mathcal{B})$ infinite appears as follows.

Take $f_{i} \in \pi_{0}\left(\mathcal{N}_{i}^{\bullet}\right), i=1,2$, and let $G_{i}$ be the subgroup of $\pi_{1}(M)$ generated by $S_{f_{i}}$ and $f_{i *}\left(\pi_{1}\left(N_{i}\right)\right)$, where $S_{f_{i}}$ is the stabilizer of $f_{i}$ as in (17.5).

Theorem 7.8 If the Reidemeister set of double cosets

$$
G_{2} \backslash \pi_{1}(M) / G_{1}
$$

is infinite, then $\pi_{0}(\mathcal{B})$ is an infinite set and $\Omega_{0}(\mathcal{B})$ is an infinitely generated abelian group.

Proof Take any $u, v \in \pi_{1}(M)$ such that the elements $\left(u f_{1}, f_{2}\right)$ and $\left(v f_{1}, f_{2}\right)$ of $\pi_{0}\left(\mathcal{N}_{1}^{\bullet}\right) \times \pi_{0}\left(\mathcal{N}_{2}^{\bullet}\right)$ give us the same element of $\pi_{0}(\mathcal{B})$.

It follows from Theorem 7.4 that there exists $(\alpha, \beta, \gamma) \in \pi_{1}\left(N_{1}\right) \times \pi_{1}\left(N_{2}\right) \times$ $\pi_{1}(M)$ such that $\gamma\left(u f_{1}, f_{2}\right)(\alpha, \beta)=\left(v f_{1}, f_{2}\right)$ or

$$
\gamma u f_{1} \alpha=v f_{1} \text { in } \pi_{0}\left(\mathcal{N}_{1}^{\bullet}\right) \quad \text { and } \quad \gamma f_{2} \beta=f_{2} \text { in } \pi_{0}\left(\mathcal{N}_{2}^{\bullet}\right) \text {. }
$$

Because of Lemma 7.1 and (7.3),$\gamma f_{2} \beta=\gamma f_{2 *}(\beta) f_{2}$. So, $\gamma f_{2 *}(\beta) \in S_{f_{2}}$, and therefore $\gamma \in G_{2}$.

Similarly, $v^{-1} \gamma u f_{1} \alpha=f_{1}$, ie $v^{-1} \gamma u f_{1 *}(\alpha) f_{1}=f_{1}$, and hence $v^{-1} \gamma u f_{1 *}(\alpha) \in$ $S_{f_{1}}$. Thus $v^{-1} \gamma u \in G_{1}$, ie $\gamma u \in v G_{1}$, and $G_{2} u \cap v G_{1} \neq \varnothing$. 
This means that the images of $u$ and $v$ in $G_{2} \backslash \pi_{1}(M) / G_{1}$ coincide. This completes the proof.

Corollary 7.9 Let $h: \pi_{1}(M) \rightarrow H_{1}(M)$ be the Hurewicz homomorphism. Then $\pi_{0}(B)$ is infinite whenever the group $H_{1}(M) /\left(h\left(G_{1}\right)+h\left(G_{2}\right)\right)$ is infinite.

Corollary 7.10 Assume that $\pi_{1}(M)$ is a Preissman group. Let $\mathcal{N}_{1} \times \mathcal{N}_{2}$ be such that for $\left(f_{1}, f_{2}\right) \in \mathcal{N}_{1} \times \mathcal{N}_{2}$ the subgroups $f_{1 *}\left(\pi_{1}\left(N_{1}\right)\right), f_{2 *}\left(\pi_{1}\left(N_{2}\right)\right) \subset$ $\pi_{1}(M)$ are both nontrivial and such that $H_{1}(M) /\left(f_{1 *}\left(H_{1}\left(N_{1}\right)\right)+f_{2 *}\left(H_{1}\left(N_{2}\right)\right)\right)$ is infinite. Then $\pi_{0}(\mathcal{B})$ is infinite and hence $\Omega_{0}(\mathcal{B})$ is an infinitely generated abelian group.

Proof It suffices to prove that each of the groups $f_{i *}\left(\pi_{1}\left(\mathcal{N}_{i}\right)\right), i=1,2$, is a subgroup of finite index in $G_{i}$. For sake of simplicity, we fix $i$ and denote $G_{i}$ and $f_{i *}\left(\pi_{1}\left(\mathcal{N}_{i}\right)\right)$ by $G$ and $H$, respectively, and prove that the index $[G: H]$ is finite.

Case 1 Assume that the group $H$ is abelian. Then since $\pi_{1}(M)$ is a Preissman group, we conclude that the centralizer $Z H$ of $H$ is a cyclic subgroup which contains $H$. Since $H \neq\{e\}, H$ is a subgroup of finite index in $Z H$. Thus, we are done since, by Proposition [7.3] $S_{f} \subset Z H$ and so $G \subset Z H$.

Case 2 Assume that the group $H$ is non-abelian. Then, by Proposition 6.3, $Z H=\{e\}$. Thus, by Proposition [7.3, $S_{f}=\{e\}$, ie $G=H$.

\section{The pairing $\mu_{i, j}$ as an obstruction for double point elimination and as a splitting of the intersection pairing}

In this section we do not assume that $\operatorname{dim} N_{1}^{n_{1}}+\operatorname{dim} N_{2}^{n_{2}}=\operatorname{dim} M^{m}$.

A classical question is: given two $C^{\infty}$ maps $f_{1}: N_{1} \rightarrow M$ and $f_{2}: N_{2} \rightarrow M$, can we make their images disjoint by homotopies of $f_{1}$ and $f_{2}$ ? The necessary condition for the existence of such homotopies is that the intersection of the homology classes $f_{1 *}\left(\left[N_{1}\right]\right)$ and $f_{2 *}\left(\left[N_{2}\right]\right)$ is zero in $H_{m-n_{1}-n_{2}}(M)$. It is wellknown that this condition is not sufficient in general.

From now on we assume that $n_{1}+n_{2}=m$ and consider the pairing

$$
\mu=\mu_{0,0}: \Omega_{0}\left(\mathcal{N}_{1}\right) \otimes \Omega_{0}\left(\mathcal{N}_{2}\right) \rightarrow \Omega_{n_{1}+n_{2}+0+0-m}(\mathcal{B})=\Omega_{0}(\mathcal{B}) .
$$


The mapping $e: \mathcal{B} \rightarrow M$ that maps $\left(\phi_{1}, \phi_{2}, \rho_{1}, \rho_{2}\right)$ to $\phi_{1} \circ \rho_{1}(\mathrm{pt})=\phi_{2} \circ$ $\rho_{2}(\mathrm{pt}) \in M$ induces the (augmentation) map $\varepsilon: \Omega_{*}(\mathcal{B}) \rightarrow \Omega_{*}(M)$. Clearly $\tau \circ \varepsilon\left(\mu\left(\left[f_{1}\right],\left[f_{2}\right]\right)\right)$ is equal to the intersection of $f_{1 *}\left(\left[N_{1}\right]\right)$ and $f_{2 *}\left(\left[N_{2}\right]\right)$ in $H_{m-n_{1}-n_{2}}(M)$, where $\tau$ is the Steenrod-Thom homomorphism.

We observe that the necessary condition for the existence of $f_{1} \in \mathcal{N}_{1}, f_{2} \in \mathcal{N}_{2}$ with $\operatorname{Im}\left(f_{1}\right) \cap \operatorname{Im}\left(f_{2}\right)=\emptyset$ is that

$$
\mu\left(\left[\bar{f}_{1}\right],\left[\bar{f}_{2}\right]\right)=0 \in \Omega_{0}(\mathcal{B}),
$$

for some (and therefore for all) $\bar{f}_{1} \in \mathcal{N}_{1}, \bar{f}_{2} \in \mathcal{N}_{2}$. Clearly this condition is much stronger than the vanishing of intersection condition. (Related results for intersections of mappings of $S^{1}$ into an oriented 2-dimensional surface were previously obtained by Turaev and Viro 33,34 .)

In fact, the following Theorem 8.1 describes a big class of manifolds $M$ where the condition

$$
\mu\left(\left[\bar{f}_{1}\right],\left[\bar{f}_{2}\right]\right)=0 \in \Omega_{0}(\mathcal{B})
$$

is necessary and sufficient for the existence of $f_{1} \in \mathcal{N}_{1}$ and $f_{2} \in \mathcal{N}_{2}$ with disjoint images.

When $N_{1}$ and $N_{2}$ are simply connected and $n_{1}+n_{2}=m$ such $f_{1} \in \mathcal{N}_{1}, f_{2} \in \mathcal{N}_{2}$ with disjoint images do exist if and only if the $\mathbb{Z}\left[\pi_{1}(M)\right]$-valued intersection index of the lifts of the mappings $\bar{f}_{1}$ and $\bar{f}_{2}$ to the universal covering $\widetilde{M} \rightarrow M$ vanishes (see Kervaire [17, for example). In the conditions of Theorem $8.1 N_{1}$ and $N_{2}$ are not assumed to be simply connected. Thus the mappings $\bar{f}_{1}$ and $\bar{f}_{2}$ can not be lifted to the universal covering and Kervaire's approach to this classical problem does not work.

Observe also that if $\mathcal{N}_{1}, \mathcal{N}_{2}$ consist of mappings homotopic to immersions, then the statement of Theorem 8.1 follows immediately from Theorem 2.2 of the work [15] of Hatcher and Quinn (see Section 2.2).

Theorem 8.1 Let $N_{1}^{n_{1}}, N_{2}^{n_{2}}, n_{1}, n_{2}>2$, be closed manifolds and let $M^{m}$ be a (not necessarily closed) manifold such that $n_{1}+n_{2}=m, \pi_{1}(M)$ is Preissman (see Definition 6.2) and $\pi_{i}(M)=0$ for $i=2, \ldots, \max \left(n_{1}, n_{2}\right)+1$. Let $\mathcal{N}_{1}, \mathcal{N}_{2}$ be connected components of the space of mappings of $N_{1}$ and of $N_{2}$ into $M$. Then $\mu: \Omega_{0}\left(\mathcal{N}_{1}\right) \otimes \Omega_{0}\left(\mathcal{N}_{2}\right) \rightarrow \Omega_{0}(\mathcal{B})=\mathbb{Z}$ is the zero pairing if and only there exists $f_{1} \in \mathcal{N}_{1}, f_{2} \in \mathcal{N}_{2}$ with $\operatorname{Im} f_{1} \cap \operatorname{Im} f_{2}=\emptyset$.

Remark 8.2 In particular, by the Preissman and Hadamard Theorems, see Theorem 6.1] and [1], $\pi_{1}(M)$ is Preissman and $\pi_{j}(M)=0, j>1$ for all closed $M$ that admit a Riemannian metric of negative sectional curvature; and any such $M$ satisfies all the conditions of Theorem 8.1 . 
Example 8.3 Here we give an example where neither Kervaire non HatcherQuinn approaches work while Theorem 8.1 implies that $f_{1}$ and $f_{2}$ can be made disjoint via a homotopy. Let $N_{1}=S^{1} \times \mathbb{R P}^{9}$, let $N_{2}$ be a 4 -dimensional manifold and let $M^{14}$ be a hyperbolic manifold. Then one can prove directly that any pair $\left(f_{1}, f_{2}\right)$ of maps $f_{1}: N_{1} \rightarrow M^{14}$ and $f_{2}: N_{2} \rightarrow M^{14}$ is homotopic to a pair with disjoint images, and so $\mu\left(f_{1}, f_{2}\right)=0$. On the other hand, the Kervaire approach is not applicable if we assume that $f_{1}$ is non-trivial on $\pi_{1}$. The Hatcher-Quinn [15] Theorem 2.2 is not applicable, since $S^{1} \times \mathbb{R P}^{9}$ is not immersible into $M^{14}$. (Since $\bar{w}_{6}\left(\mathbb{R} P^{9}\right) \neq 0$, neither $\mathbb{R P}^{9}$ nor $S^{1} \times \mathbb{R} \mathbb{P}^{9}$ are immersible into the universal cover $\mathbb{R}^{14}$ of $M^{14}$.)

Proof It is clear that if $f_{1} \in \mathcal{N}_{1}$ and $f_{2} \in \mathcal{N}_{2}$ with $\operatorname{Im} f_{1} \cap \operatorname{Im} f_{2}=\emptyset$ do exist, then $\mu$ is the zero pairing.

Let us show that if $\mu$ is the zero pairing, then such $f_{1}, f_{2}$ do exist. Take $\bar{f}_{1} \in \mathcal{N}_{1}, \bar{f}_{2} \in \mathcal{N}_{2}$ so that they are transversal to each other, and hence $\operatorname{Im} \bar{f}_{1}$ and $\operatorname{Im} \bar{f}_{2}$ intersect at isolated transverse double points. For a double point $d \in \operatorname{Im} \bar{f}_{1} \cap \operatorname{Im} \bar{f}_{2}$ we denote by $[d] \in \Omega_{0}(\mathcal{B})$ the input of this double point into $\mu\left(\left[\bar{f}_{1}\right],\left[\bar{f}_{2}\right]\right)$. The proof is via induction on the number of double points of $\operatorname{Im} \bar{f}_{1} \cap \operatorname{Im} \bar{f}_{2}$.

Take a double point $p \in \operatorname{Im} \bar{f}_{1} \cap \operatorname{Im} \bar{f}_{2}$. Since $\mu\left(\left[\bar{f}_{1}\right],\left[\bar{f}_{2}\right]\right)=0$, there exists a transversal double point $q \in \operatorname{Im} \bar{f}_{1} \cap \operatorname{Im} \bar{f}_{2}$ such that $[p]=-[q] \in \Omega_{0}(\mathcal{B})$. In particular we get that the double points $p$ and $q$ correspond to the same path connected component of $\mathcal{B}$. Put $p_{i}$ and $q_{i}$ (for $i=1,2$ ) to be, respectively, the preimages of the double points $p$ and $q$ on $N_{i}$ (for $i=1,2$ ).

Theorem 7.4 and the definition of $\mathcal{B}$ imply that there exist paths $\alpha_{i}:[0,1] \rightarrow N_{i}$ with $\alpha_{i}(0)=p_{i}$ and $\alpha_{i}(1)=q_{i}$ for $i=1,2$; and a path $\beta:[1,2] \rightarrow M$ with $\beta(1)=q, \beta(2)=p$ such that the loop $\bar{f}_{i}\left(\alpha_{i}\right) \beta$ acts trivially on the pointed homotopy class of the mapping $\left(N_{i}, p_{i}\right) \rightarrow(M, p)$ for $i=1,2$. Thus $\bar{f}_{1}\left(\alpha_{1}\right) \beta \in$ $\pi_{1}(M, p)$ commutes with all the elements of $\operatorname{Im}\left(\bar{f}_{1 *}: \pi_{1}\left(N_{1}\right) \rightarrow \pi_{1}(M)\right)$.

If $\operatorname{Im} \bar{f}_{1} \subset \pi_{1}(M)$ is an infinite cyclic group or a trivial group, then, since $\pi_{j}(M)=0$ for $j=2, \ldots, \max \left(n_{1}, n_{2}\right)+1$, the elementary obstruction theory implies that $\bar{f}_{1}$ is homotopic to a mapping that passes through a mapping $S^{1} \rightarrow$ $M$. Since $n_{1}+n_{2}=m$ and $n_{1}>2$ we get that after the small perturbation the mapping $S^{1} \rightarrow M$ does not pass through $\operatorname{Im} \bar{f}_{2}$ and thus $\bar{f}_{1}$ is homotopic to a mapping that has disjoint image with $\bar{f}_{2}$.

If $\operatorname{Im} \bar{f}_{1 *} \subset \pi_{1}(M)$ is a group which is not trivial and is not infinite cyclic, then, since $\pi_{1}(M)$ is Preissman (see Definition [6.2), $\bar{f}_{1}\left(\alpha_{1}\right) \beta=1 \in \pi_{1}(M, p)$. 
Similarly, we get that either $\bar{f}_{2}$ is homotopic to mapping passing through a mapping $S^{1} \rightarrow M$, and hence it is homotopic to a mapping with the image disjoint from $\operatorname{Im} \bar{f}_{1}$; or $\bar{f}_{2}\left(\alpha_{2}\right) \beta$ is also trivial in $\pi_{1}(M)$. Thus the paths $\bar{f}_{1}\left(\alpha_{1}\right)$ and $\bar{f}_{2}\left(\alpha_{2}\right)$ can be assumed to be homotopic. Thus $\bar{f}_{1}\left(\alpha_{1}\right)\left(\bar{f}_{2}\left(\alpha_{2}\right)\right)^{-1}=1 \in$ $\pi_{1}(M)$ and this loop bounds a disk $D \subset M$.

For dimension reasons $D$ can be assumed to be embedded and $\operatorname{Int} D$ can be assumed to be disjoint from $\operatorname{Im} \bar{f}_{1}$ and $\operatorname{Im} \bar{f}_{2}$. Since $[p]=-[q] \in \Omega_{0}(\mathcal{B})$, we get that the signs of the intersection points $p$ and $q$ of $\operatorname{Im} \bar{f}_{1} \cap \operatorname{Im} \bar{f}_{2}$ are opposite. Thus we can apply the Whitney trick to the points $p$ and $q$ and the disk $D$ and cancel the double points. Since $D$ was assumed to be embedded and $\operatorname{Int} D$ was assumed to be disjoint from $\operatorname{Im} \bar{f}_{1}$ and $\operatorname{Im} \bar{f}_{2}$, no new double points of $\operatorname{Im} \bar{f}_{1} \cap \operatorname{Im} \bar{f}_{2}$ will appear during the Whitney trick. Thus we managed to decrease the number of double points of $\operatorname{Im} \bar{f}_{1} \cap \operatorname{Im} \bar{f}_{2}$ and we proceed by induction.

\section{References}

[1] V I Arnol'd, Plane curves, their invariants, perestroikas and classifications, from: "Singularities and bifurcations", Adv. Soviet Math. 21, Amer. Math. Soc. Providence, RI (1994) 33-91 MathReview

[2] V I Arnol'd, Invariants and perestroikas of fronts on a plane, Trudy Mat. Inst. Steklov. 209 (1995) 14-64 MathReview

[3] V I Arnol'd, Mathematical methods of classical mechanics, Graduate Texts in Mathematics 60, Springer, New York (1989) MathReview

[4] M Chas, D Sullivan, String Topology, arXiv:math.GT/9911159

[5] M Chas, D Sullivan, Closed string operators in topology leading to Lie bialgebras and higher string algebra, from: "The legacy of Niels Henrik Abel", Springer, Berlin (2004) 771-784 MathReview

[6] V Chernov, Framed knots in 3-manifolds and Affine Self-linking numbers, J. Knot Theory Ramifications 14 (2005) 791-819

[7] V Chernov, Yu B Rudyak, Affine linking numbers and causality relations for wave fronts, arXiv:math.GT/0207219

[8] V Chernov, Yu B Rudyak, Affine winding numbers and front propagation, arXiv:math.GT/0301117

[9] V Chernov, YuB Rudyak, Algebraic Structures on Generalized Strings, arXiv:math.GT/0306140

[10] V Chernov, Yu B Rudyak, Toward the General Theory of Affine Linking Numbers, arXiv:math.GT/0302295 
[11] M P do Carmo, Riemannian geometry, Mathematics: Theory \& Applications, Birkhäuser Boston Inc. Boston, MA (1992) MathReview

[12] W M Goldman, Invariant functions on Lie groups and Hamiltonian flows of surface group representations, Invent. Math. 85 (1986) 263-302 MathReview

[13] M N Goussarov, Talk at the Rokhlin Seminar, Steklov Institute for Mathematics, St Petersburg, Russia, 12th January 1987

[14] M N Goussarov, A new form of the Conway-Jones polynomial of oriented links, Zap. Nauchn. Sem. Leningrad. Otdel. Mat. Inst. Steklov. (LOMI) 193 (1991) 4-9, 161 MathReview

[15] A Hatcher, F Quinn, Bordism invariants of intersections of submanifolds, Trans. Amer. Math. Soc. 200 (1974) 327-344 MathReview

[16] U Kaiser, Link theory in manifolds, Lecture Notes in Mathematics 1669, Springer, Berlin (1997) MathReview

[17] M A Kervaire, Le théorème de Barden-Mazur-Stallings, Comment. Math. Helv. 40 (1965) 31-42 MathReview

[18] U Koschorke, Nielsen coincidence theory in arbitrary codimensions, arXiv:math.AT/0408044

[19] U Koschorke, Linking and coincidence invariants, Fund. Math. 184 (2004) 187-203 MathReview

[20] R J Low, Twistor linking and causal relations, Classical Quantum Gravity 7 (1990) 177-187 MathReview

[21] R J Low, Celestial spheres, light cones, and cuts, J. Math. Phys. 34 (1993) 315-319 MathReview

[22] R J Low, Twistor linking and causal relations in exterior Schwarzschild space, Classical Quantum Gravity 11 (1994) 453-456 MathReview

[23] J Natário, P Tod, Linking, Legendrian linking and causality, Proc. London Math. Soc. (3) 88 (2004) 251-272 MathReview

[24] A Preissman, Quelques propriétés globales des espaces de Riemann, Comment. Math. Helv. 15 (1943) 175-216 MathReview

[25] Y B Rudyak, On Thom spectra, orientability, and cobordism, Springer Monographs in Mathematics, Springer, Berlin (1998) MathReview

[26] R Schneiderman, Algebraic linking numbers of knots in 3-manifolds, Algebr. Geom. Topol. 3 (2003) 921-968 MathReview

[27] E H Spanier, Algebraic topology, Springer, New York (1981) MathReview

[28] R E Stong, Notes on cobordism theory, Mathematical notes, Princeton University Press, Princeton, N.J. (1968) MathReview

[29] R M Switzer, Algebraic topology: homotopy and homology, Grundlehren der mathematischen Wissenschaften 212, Springer, New York (1975) MathReview 
[30] S L Tabachnikov, Calculation of the Bennequin invariant of a Legendre curve from the geometry of its wave front, Funktsional. Anal. i Prilozhen. 22 (1988) 89-90 MathReview

[31] R Thom, Quelques propriétés globales des variétés différentiables, Comment. Math. Helv. 28 (1954) 17-86 MathReview

[32] V G Turaev, Skein quantization of Poisson algebras of loops on surfaces, Ann. Sci. École Norm. Sup. (4) 24 (1991) 635-704 MathReview

[33] V G Turaev, Intersections of loops in two-dimensional manifolds, Mat. Sb. 106(148) (1978) 566-588 MathReview

[34] V G Turaev, OY Viro, Intersection of loops in two-dimensional manifolds II: Free loops, Mat. Sb. (N.S.) 121(163) (1983) 359-369 MathReview

[35] V A Vassiliev, Cohomology of knot spaces, from: "Theory of singularities and its applications", Adv. Soviet Math. 1, Amer. Math. Soc., Providence, RI (1990) 23-69 MathReview

[36] A A Voronov, Notes on universal algebra, from: "Graphs and patterns in mathematics and theoretical physics", Proc. Sympos. Pure Math. 73, Amer. Math. Soc. Providence, RI (2005) 81-103 MathReview 